\title{
Coupled Fixed Point Theorems with New Implicit Relations and an Application
}

\author{
G. V. R. Babu ${ }^{1}$ and P. D. Sailaja ${ }^{1,2}$ \\ ${ }^{1}$ Department of Mathematics, Andhra University, Visakhapatnam 530 003, India \\ ${ }^{2}$ Department of Mathematics, Lendi Institute of Engineering and Technology, Vizianagaram 535 005, India \\ Correspondence should be addressed to G. V. R. Babu; gvr_babu@hotmail.com
}

Received 28 June 2014; Accepted 1 October 2014; Published 11 November 2014

Academic Editor: Hagen Neidhardt

Copyright (c) 2014 G. V. R. Babu and P. D. Sailaja. This is an open access article distributed under the Creative Commons Attribution License, which permits unrestricted use, distribution, and reproduction in any medium, provided the original work is properly cited.

\begin{abstract}
We introduce two new classes of implicit relations $\mathcal{S}$ and $\mathcal{S}^{\prime}$ where $\mathcal{S}^{\prime}$ is a proper subset of $\mathcal{S}$, and these classes are more general than the class of implicit relations defined by Altun and Simsek (2010). We prove the existence of coupled fixed points for the maps satisfying an implicit relation in $\mathcal{S}$. These coupled fixed points need not be unique. In order to establish the uniqueness of coupled fixed points we use an implicit relation $\mathcal{S}^{\prime}$, where $\mathcal{S}^{\prime} \subset \mathcal{S}$. Our results extend the fixed point theorems on ordered metric spaces of Altun and Simsek (2010) to coupled fixed point theorems and generalize the results of Gnana Bhaskar and Lakshimantham (2006). As an application of our results, we discuss the existence and uniqueness of solution of Fredholm integral equation.
\end{abstract}

\section{Introduction}

Existence of fixed point theorems in partially ordered metric spaces with a contractive condition has been considered by several authors (see [1-6]). Guo and Lakshmikantham [7] introduced mixed monotone operators. Gnana Bhaskar and Lakshmikantham [8] established the existence of coupled fixed points of mappings satisfying mixed monotone property in partially ordered metric spaces. Later, Lakshmikantham and Ciric [9] extended this property to two maps by introducing mixed $g$-monotone property and established the existence of coupled coincidence point and coupled common fixed points for a pair of commuting maps.

Choudhury and Kundu [10] extended the existence of coupled coincidence and coupled common fixed points for a pair of noncommuting maps, particularly for a pair of compatible maps.

Definition 1 (see [7]). Let $X$ be a nonempty set. An element $(x, y)$ in $X \times X$ is called a coupled fixed point of the mapping $F: X \times X \rightarrow X$ if $x=F(x, y)$ and $y=F(y, x)$. A point $x \in X$ is called a fixed point of $F$ if $F(x, x)=x$.

Definition 2 (see [7]). Let $(X, \preceq)$ be a partially ordered set and $F: X \times X \rightarrow X$ be a mapping. We say that $F$ satisfies mixed monotone property if $F(x, y)$ is monotone nondecreasing in $x$ and monotone nonincreasing in $y$; that is, for any $x, y \in X$ :

$$
\begin{aligned}
& x_{1}, x_{2} \in X, \quad x_{1} \preceq x_{2} \Longrightarrow F\left(x_{1}, y\right) \preceq F\left(x_{2}, y\right), \\
& y_{1}, y_{2} \in X, \quad y_{1} \preceq y_{2} \Longrightarrow F\left(x, y_{1}\right) \succeq F\left(x, y_{2}\right) .
\end{aligned}
$$

Theorem 3 (see $[8]$ ). Let $(X, \preceq)$ be a partially ordered set and suppose that $d$ is a metric on $X$ such that $(X, d)$ is a complete metric space. Let $F: X \times X \rightarrow X$ be a mapping satisfying mixed monotone property. Assume that there exists a $k \in[0,1)$ with

$$
\begin{array}{r}
d(F(x, y), F(u, v)) \leq \frac{k}{2}[d(x, u)+d(y, v)] \\
\text { for each } x \geq u, y \leq v .
\end{array}
$$

Suppose that either $F$ is continuous or the following conditions hold in $X$ :

(i) if a nondecreasing sequence $\left\{x_{n}\right\} \subseteq X$ with $x_{n} \rightarrow x$, then $x_{n} \preceq x$ for all $n$ and

(ii) if a nonincreasing sequence $\left\{y_{n}\right\} \subseteq X$ with $y_{n} \rightarrow y$, then $y_{n} \geq y$ for all $n$.

If there exist $x_{0}, y_{0} \in X$ such that $x_{0} \preceq F\left(x_{0}, y_{0}\right)$ and $y_{0} \succeq$ $F\left(y_{0}, x_{0}\right)$ then $F$ has a coupled fixed point. 
In 2011, Luong and Thuan [11] proved the following coupled fixed point theorem.

Theorem 4 (see $[11])$. Let $(X, \preceq)$ be a partially ordered set and suppose that $d$ is a metric on $X$ such that $(X, d)$ is a complete metric space. Let $F: X \times X \rightarrow X$ be a mapping satisfying mixed monotone property on $X$ and there exist $x_{0}, y_{0} \in X$ such that $x_{0} \preceq F\left(x_{0}, y_{0}\right)$ and $y_{0} \succeq F\left(y_{0}, x_{0}\right)$. Suppose that there exists a $\psi:[0, \infty) \rightarrow[0, \infty)$ with $\lim _{t \rightarrow r^{+}} \psi(t)>0$ for all $r>0$ and $\lim _{t \rightarrow 0^{+}} \psi(t)=0$ such that

$$
\begin{aligned}
d(F(x, y), F(u, v)) \leq & \frac{d(x, u)+d(y, v)}{2} \\
& -\psi\left(\frac{d(x, u)+d(y, v)}{2}\right),
\end{aligned}
$$

for each $x, y, u, v \in X, x \geq u$ and $y \preceq v$. Suppose that either

(a) $F$ is continuous or

(b) X has the following property:

(i) if a nondecreasing sequence $\left\{x_{n}\right\} \subseteq X$ with $x_{n} \rightarrow$ $x$, then $x_{n} \preceq x$ for all $n$ and

(ii) if a nonincreasing sequence $\left\{y_{n}\right\} \subseteq X$ with $y_{n} \rightarrow$ $y$, then $y_{n} \geq y$ for all $n$.

Then $F$ has a coupled fixed point.

In Section 2 of this paper, we introduce two new classes of implicit relations $\mathcal{S}$ and $\mathcal{S}^{\prime}$ where $\mathcal{S}^{\prime}$ is a proper subset of $\mathcal{S}$, and these classes are more general than the class of implicit relations defined by Altun and Simsek [12]. In Section 3, we prove the existence of coupled fixed points for the maps satisfying an implicit relation in $\delta$. These coupled fixed points need not be unique (Example 17). In order to establish the uniqueness of coupled fixed points we use an implicit relation $\mathcal{S}^{\prime}$, where $\mathcal{S}^{\prime} \subset \mathcal{S}$. Our results extend the fixed point theorems on ordered metric spaces of Altun and Simsek [12] to coupled fixed point theorems and generalize the results of Gnana Bhaskar and Lakshimantham [8]. In Section 4, as an application to results of Section 3, we discuss the existence and uniqueness of solution of Fredholm integral equation.

\section{Implicit Relations}

In 1999, Popa [13] introduced the idea of the class $\mathfrak{F}$ of implicit functions to prove the existence of fixed points as follows.

Let $R_{+}$denote the set of all nonnegative real numbers. Let $\mathfrak{F}$ be the family of real lower semicontinuous functions $F$ : $\left(R_{+}\right)^{6} \rightarrow R$ satisfying the following conditions:

$\left(F_{1}\right): F$ is nonincreasing in fifth and sixth variables;

$\left(F_{2}\right)$ : there exists $h \in(0,1)$ such that for every $u, v \geq 0$ with

$$
\begin{aligned}
F_{2 a}: F(u, v, v, u, u+v, 0) \leq 0 \text { or } \\
F_{2 b}: F(u, v, u, v, 0, u+v) \leq 0 \\
\quad \text { we have } u \leq h v ;
\end{aligned}
$$$$
\left(F_{3}\right): F(u, u, 0,0, u, u)>0, \forall u>0 .
$$

Many authors, namely, Altun and Simsek [12], Altun and Turkoglu [14, 15], Imdad et al. [16], Popa [17], Popa and Mocanu [18], Sharma and Deshpande [19], and Turkoglu and Altun [20], continued the study of the existence of fixed points in this direction. The purpose of using an implicit relation is that the condition involving implicit relation generalizes many contraction conditions, which in turn generalizes many existing results. In 2010, Altun and Simsek [12] defined a new class of implicit relations as follows and established a fixed point theorem in ordered metric spaces.

Let $\mathscr{T}$ be the set of all continuous functions $T:\left(R_{+}\right)^{6} \rightarrow$ $R$ satisfying the following conditions:

(T1): $T\left(t_{1}, \ldots, t_{6}\right)$ is nonincreasing in variables $t_{2}, t_{3}, \ldots, t_{6}$;

(T2): there exists a right continuous function $f: R_{+} \rightarrow R_{+}$, $f(0)=0, f(t)<t$ for $t>0$, such that for $u, v \geq 0$

$$
\begin{aligned}
& T(u, v, u, v, 0, u+v) \leq 0 \text { or } T(u, v, 0,0, v, v) \leq 0 \\
& \text { implies } u \leq f(v)
\end{aligned}
$$$$
\text { (T3): } T(u, 0, u, 0,0, u)>0 \text { for } u>0 \text {. }
$$

Theorem 5 (see [12]). Let $(X, \preceq)$ be a partially ordered set and suppose that $d$ is a metric on $X$ such that $(X, d)$ is a complete metric space. Let $F: X \rightarrow X$ be a nondecreasing mapping such that, for all $x, y \in X$ with $y \preceq x$,

$$
\begin{aligned}
& T(d(F x, F y), d(x, y), d(x, F x), \\
& \quad d(y, F y), d(x, F y), d(y, F x)) \leq 0,
\end{aligned}
$$

where $T \in \mathscr{T}$. Also, suppose that either $F$ is continuous or the following condition holds: "if a nondecreasing sequence $\left\{x_{n}\right\} \rightarrow$ $x$, then $x_{n} \preceq x$ for all $n$ ”.

If there exists an $x_{0} \in X$ with $x_{0} \preceq F\left(x_{0}\right)$, then $F$ has a fixed point.

We now define a more general class of implicit relations than that of [12] as follows.

Let $\mathcal{S}$ be the set of all continuous functions $T:\left(R_{+}\right)^{6} \rightarrow$ $R$ satisfying the following conditions:

$$
\left(T_{1}\right): T\left(t_{1}, \ldots, t_{6}\right) \text { is nonincreasing in variables } t_{5}, t_{6} \text {; }
$$

$\left(T_{2}\right)$ : there exists a mapping $f: R_{+} \rightarrow R_{+}, f(t)<t$ for $t>0$, such that for $u, v \geq 0$

$$
\begin{aligned}
& T(u, v, u, v, 0, u+v) \leq 0 \quad \text { or } \quad T(u, v, 0,0, v, v) \leq 0, \\
& \text { implies } u \leq f(v)
\end{aligned}
$$$$
\left(T_{3}\right): T(u, 0, u, 0,0, u)>0 \text { for } u>0 .
$$

Here we observe that $\mathscr{T} \subseteq \mathcal{S}$.

Example 6. $T\left(t_{1}, \ldots, t_{6}\right)=t_{1}-\left[a t_{2}+b t_{3}+c t_{4}+d t_{5}+e t_{6}\right]$, where $a, b, c, d, e \geq 0$ and $a+b+c+d+2 e<1$. 
Clearly $\left(T_{1}\right)$ holds. Let $T(u, v, u, v, 0, u+v)=u-(a v+$ $b u+c v+e(u+v))=(1-b-e) u-(a+c+e) v \leq 0$. Thus $u \leq((a+c+e) /(1-b-e)) v$. Let $T(u, v, 0,0, v, v)=$ $u-(a v+d v+e v)=u-(a+d+e) v \leq 0$. Thus $u \leq$ $(a+d+e) v$. Now define $f: R_{+} \rightarrow R_{+}$by $f(t)=k t$, where $k=\max \{((a+c+e) /(1-b-e)), a+d+e\}<1, f(t)<t$ for $t>0$. Hence $u \leq f(v)$. Also, $T(u, 0, u, 0,0, u)=u-(b u+e u)=$ $(1-b-e) u>0$ for $u>0$. Therefore $T \in \mathcal{S}$.

Example 7. $T\left(t_{1}, \ldots, t_{6}\right)=t_{1}-\varphi\left(\max \left\{t_{2}, t_{3}, t_{4},(1 / 2)\left(t_{5}+t_{6}\right)\right\}\right)$, where $\varphi: R_{+} \rightarrow R_{+}$is a continuous map with $\varphi(0)=0$, $\varphi(t)<t$ for $t>0$.

Clearly $\left(T_{1}\right)$ holds. Let $u>0$ and $T(u, v, u, v, 0, u+v)=u-$ $\varphi(\max \{v, u, v,(1 / 2)(u+v)\})=u-\varphi(\max \{u, v\}) \leq 0$. If $u \geq v$ then $u-\varphi(u) \leq 0$, which is a contradiction. Hence $u<v$ and hence $u \leq \varphi(v)$. Let $u>0$ and $T(u, v, 0,0, v, v)=u-\varphi(v) \leq 0$; that is, $u \leq \varphi(v)$. If $u=0$ then clearly $u \leq \varphi(v)$. Thus $\left(T_{2}\right)$ is satisfied with $f=\varphi$. Also, $T(u, 0, u, 0,0, u)=u-\varphi(u)>0$ for $u>0$. Therefore $T \in \mathcal{S}$.

Example 8. $T\left(t_{1}, \ldots, t_{6}\right)=t_{1}-\left(t_{2}-\varphi\left(t_{2}\right)\right)$, where $\varphi: R_{+} \rightarrow$ $R_{+}$is a continuous map with $\varphi(t)=0$ if and only if $t=0$, $\varphi(t)<t$ for $t>0$.

Clearly $\left(T_{1}\right)$ holds. Let $T(u, v, u, v, 0, u+v)=u-(v-$ $\varphi(v)) \leq 0$. Let $T(u, v, 0,0, v, v)=u-(v-\varphi(v)) \leq 0$; that is, $u \leq v-\varphi(v)$. Thus $u \leq f(v)$ with $f: R_{+} \rightarrow R_{+}$by $f(t)=$ $t-\varphi(t)$. Hence $\left(T_{2}\right)$ is satisfied. Also, $T(u, 0, u, 0,0, u)=u-$ $(u-\varphi(u))=\varphi(u)>0$ for $u>0$. Hence $T \in \mathcal{S}$.

Example 9. $T\left(t_{1}, \ldots, t_{6}\right)=t_{1}-k t_{2}$, where $0 \leq k<1$.

Clearly $\left(T_{1}\right)$ holds. Let $T(u, v, u, v, 0, u+v)=u-k v \leq 0$; that is, $u \leq k v$. Let $T(u, v, 0,0, v, v)=u-k v \leq 0$; that is, $u \leq k v$. Thus, $u \leq f(v)$ with $f(t)=k t$. Hence, $T_{2}$ is satisfied. Also, $T(u, 0, u, 0,0, u)=u>0$ for $u>0$. Thus $T \in \mathcal{S}$.

Example 10. $T\left(t_{1}, \ldots, t_{6}\right)=t_{1}-\varphi\left(t_{2}\right)$, where $\varphi: R_{+} \rightarrow R_{+}$is a continuous map with $\varphi(0)=0, \varphi(t)<t$ for $t>0$.

Clearly $\left(T_{1}\right)$ holds. Let $T(u, v, u, v, 0, u+v)=u-\varphi(v) \leq 0$; that is, $u \leq \varphi(v)$. Let $T(u, v, 0,0, v, v)=u-\varphi(v) \leq 0$; that is, $u \leq \varphi(v)$. Thus $u \leq f(v)$ with $f=\varphi$. Hence $\left(T_{2}\right)$ is satisfied. Also, $T(u, 0, u, 0,0, u)=u-\varphi(0)=u>0$ for $u>0$. Hence $T \in \mathcal{S}$.

\section{Example 11.}

$$
\begin{array}{r}
T\left(t_{1}, \ldots, t_{6}\right)=t_{1}-\alpha \frac{t_{4} t_{5} t_{6}}{t_{2}+t_{3}+1}, \\
\text { where } 0 \leq \alpha<1 .
\end{array}
$$

$T$ is nonincreasing in $t_{5}$ and $t_{6}$ but not in any of $t_{2}, t_{3}$. Let $u>0, T(u, v, u, v, 0, u+v)=u-\alpha(0)=u<0$, a contradiction. Now let $T(u, v, 0,0, v, v)=u-\alpha(0)=u<0$, a contradiction. If $u=0$ then clearly $u \leq \alpha v$. Thus $\left(T_{2}\right)$ is satisfied. Also, $T(u, 0, u, 0,0, u)=u>0$ for $u>0$. Thus $T \in \mathcal{S}$. But $T$ does not belong to $\mathscr{T}$.

In view of Example 11, the class of implicit functions $\mathcal{S}$ is larger than that of $\mathscr{T}$ introduced by Altun and Simsek [12] and we use the implicit relation involving implicit function of $\mathcal{S}$ to prove our main results.
In this paper, we prove a coupled fixed point theorem (Theorem 16) using an implicit relation in $\mathcal{S}$. In order to obtain the uniqueness of coupled fixed point we define another class $\mathcal{S}^{\prime}$ of implicit relations as follows.

Let $\mathcal{S}^{\prime}$ be the set of all continuous functions $T:\left(R_{+}\right)^{6} \rightarrow$ $R$ satisfying the following conditions:

$\left(T_{1}^{\prime}\right): T\left(t_{1}, \ldots, t_{6}\right)$ is nonincreasing in variables $t_{3}, t_{5}, t_{6}$;

$\left(T_{2}^{\prime}\right)$ : there exists a mapping $f: R_{+} \rightarrow R_{+}, f(t)<t$ for $t>0$, such that, for each $u, v \geq 0$,

$$
\begin{gathered}
T(u, v, u, v, 0, u+v) \leq 0 \quad \text { or } T(u, v, 0,0, v, v) \leq 0 \\
\text { or } T(u, v, u+v, 0, v, u) \leq 0
\end{gathered}
$$

implies $u \leq f(v)$;

$\left(T_{3}^{\prime}\right): T(u, 0, u, 0,0, u)>0$ for $u>0$.

Here we observe that $\mathcal{S}^{\prime} \subseteq \mathcal{S}$.

All functions in Examples 8-10 are in $\mathcal{S}^{\prime}$. Some more examples in this direction are as follows.

Example 12. $T\left(t_{1}, \ldots, t_{6}\right)=t_{1}-\left[a t_{2}+b t_{3}+c t_{4}+d t_{5}+e t_{6}\right]$, where $a, b, c, d, e \geq 0$ and $a+b+c+d+2 e<1$ and $b \leq c$.

Clearly $\left(T_{1}^{\prime}\right)$ holds. Let $T(u, v, u, v, 0, u+v)=u-(a v+$ $b u+c v+e(u+v))=(1-b-e) u-(a+c+e) v \leq 0$. Thus $u \leq((a+c+e) /(1-b-e)) v$. Let $T(u, v, 0,0, v, v)=u-(a v+$ $d v+e v)=u-(a+d+e) v \leq 0$. Thus $u \leq(a+d+e) v$. Now let $T(u, v, u+v, 0, v, u)=u-[a v+b(u+v)+d v+e u] \leq 0$. This implies $u \leq((a+c+d) /(1-b-e)) v$. Now define $f: R_{+} \rightarrow$ $R_{+}$by $f(t)=k t$, where $k=\max \{((a+c+e) /(1-b-e)), a+$ $d+e,((a+c+d) /(1-b-e))\}<1, f(t)<t$ for $t>0$. Hence $u \leq f(v)$. Also, $T(u, 0, u, 0,0, u)=u-(b u+e u)=(1-b-e) u>$ 0 for $u>0$. Therefore $T \in \mathcal{S}^{\prime}$.

Example 13. $T\left(t_{1}, \ldots, t_{6}\right)=t_{1}-\varphi\left(\max \left\{t_{2}, t_{3} / 2, t_{4},(1 / 2)\left(t_{5}+\right.\right.\right.$ $\left.\left.t_{6}\right)\right\}$ ), where $\varphi: R_{+} \rightarrow R_{+}$with $\varphi(0)=0, \varphi(t)<t$ for $t>0$.

Clearly $\left(T_{1}^{\prime}\right)$ holds. Let $u>0$ and $T(u, v, u, v, 0, u+v)=$ $u-\varphi(\max \{v, u / 2, v,(1 / 2)(u+v)\})=u-\varphi(\max \{u, v\}) \leq 0$. If $u \geq v$, then $u-\varphi(u) \leq 0$, which is a contradiction. Hence $u<v$ and hence $u \leq \varphi(v)$. Let $u>0$ and $T(u, v, 0,0, v, v)=$ $u-\varphi(v) \leq 0$; that is, $u \leq \varphi(v)$. Let $u>0$ and $T(u, v, u+$ $v, 0, v, u)=u-\varphi(\max \{v, u,(u+v) / 2,0,(1 / 2)(u+v)\})=u-$ $\varphi(\max \{u, v\}) \leq 0$. If $u \geq v$, then $u-\varphi(u) \leq 0$, which is a contradiction. Hence $u<v$ and hence $u \leq \varphi(v)$. If $u=0$, then clearly $u \leq \varphi(v)$. Thus $\left(T_{2}^{\prime}\right)$ is satisfied with $f=\varphi$. Also, $T(u, 0, u, 0,0, u)=u-\varphi(u)>0$ for $u>0$. Therefore, $T \in \mathcal{S}^{\prime}$. of $\mathcal{S}$.

The following examples suggest that $\delta^{\prime}$ is a proper subset

Example 14. $T\left(t_{1}, \ldots, t_{6}\right)=t_{1}-\varphi\left(\max \left\{t_{2}, t_{3}, t_{4},(1 / 2)\left(t_{5}+\right.\right.\right.$ $\left.\left.t_{6}\right)\right\}$ ), where $\varphi: R_{+} \rightarrow R_{+}$defined by $\varphi(t)=t / 2$. In view of Example 7, $T \in \mathcal{S}$. But $T$ is not in $\mathcal{S}^{\prime}$ for let $u, v>0, v \leq u$ and suppose that $T(u, v, u+v, 0, v, u)=u-\varphi(\max \{v, u+v, 0,(u+$ $v) / 2\})=u-\varphi(u+v)=u-(u+v) / 2 \leq 0$. This implies $u \leq v$. Therefore $u=v$. Hence, there cannot exist a function $f: R_{+} \rightarrow R_{+}$with $f(t)<t$ for $t>0$ such that $u \leq f(v)$. Hence $\mathcal{S}^{\prime} \subset \mathcal{S}$. 
Example 15. Let us consider $T$ as in Example 11. Then $T \in \mathcal{S}$. But $T$ does not belong to $\mathcal{S}^{\prime}$, since $T$ is not nonincreasing in $t_{3}$, so that $\mathcal{S}^{\prime}$ is a proper subset of $\mathcal{S}$.

\section{Existence of Coupled Fixed Points Using a New Implicit Relation}

Theorem 16. Let $(X, \preceq)$ be a partially ordered set and suppose that $d$ is a metric on $X$ such that $(X, d)$ is a complete metric space. Let $F: X \times X \rightarrow X$ be a mapping satisfying mixed monotone property and suppose that there exists $T \in \mathcal{S}$ such that

$$
\begin{aligned}
T( & \frac{d(F(x, y), F(u, v))+d(F(y, x), F(v, u))}{2}, \\
& \frac{d(x, u)+d(y, v)}{2}, \frac{d(x, F(x, y))+d(y, F(y, x))}{2}, \\
& \frac{d(u, F(u, v))+d(v, F(v, u))}{2}, \\
& \frac{d(x, F(u, v))+d(y, F(v, u))}{2}, \\
& \left.\frac{d(u, F(x, y))+d(v, F(y, x))}{2}\right) \leq 0
\end{aligned}
$$

for each $x, y, u, v \in X$ with $x \geq u$ and $y \preceq v$.

Suppose that either

(a) $F$ is continuous or

(b) X has the following property:

(i) if a nondecreasing sequence $\left\{x_{n}\right\} \subseteq X$ with $x_{n} \rightarrow$ $x$, then $x_{n} \preceq x$ for all $n$ and

(ii) if a nonincreasing sequence $\left\{y_{n}\right\} \subseteq X$ with $y_{n} \rightarrow$ $y$, then $y_{n} \geq y$ for all $n$.

If there exist $x_{0}, y_{0} \in X$ such that $x_{0} \preceq F\left(x_{0}, y_{0}\right)$ and $y_{0} \succeq$ $F\left(y_{0}, x_{0}\right)$ then $F$ has a coupled fixed point.

Proof. Suppose that there exists $\left(x_{0}, y_{0}\right) \in X \times X$ such that $x_{0} \preceq F\left(x_{0}, y_{0}\right)$ and $y_{0} \succeq F\left(y_{0}, x_{0}\right)$. Choose $x_{1}, y_{1} \in X$ such that $x_{1}=F\left(x_{0}, y_{0}\right)$ and $y_{1}=F\left(y_{0}, x_{0}\right)$. Then $x_{0} \preceq x_{1}$ and $y_{0} \geq y_{1}$. Now choose $x_{2}, y_{2} \in X$ such that $x_{2}=F\left(x_{1}, y_{1}\right)$ and $y_{2}=F\left(y_{1}, x_{1}\right)$. Since $F$ has mixed monotone property, we get

$$
\begin{aligned}
& x_{1}=F\left(x_{0}, y_{0}\right) \preceq F\left(x_{1}, y_{1}\right)=x_{2}, \\
& y_{1}=F\left(y_{0}, x_{0}\right) \succeq F\left(y_{1}, x_{1}\right)=y_{2} .
\end{aligned}
$$

Continuing this process, we obtain sequences $\left\{x_{n}\right\}$ and $\left\{y_{n}\right\}$ in $X$ such that $x_{n+1}=F\left(x_{n}, y_{n}\right), y_{n+1}=F\left(y_{n}, x_{n}\right)$ for $n=$ $0,1,2, \ldots$ and

$$
x_{0} \preceq x_{1} \preceq x_{2} \preceq \cdots, \quad y_{0} \geq y_{1} \geq y_{2} \geq \cdots .
$$

Let $x_{n+1}=F\left(x_{n}, y_{n}\right)=F^{n}\left(x_{0}, y_{0}\right)=F\left(F^{n-1}\left(x_{0}, y_{0}\right), F^{n-1}\left(y_{0}\right.\right.$, $\left.x_{0}\right)$ ) and $y_{n+1}=F\left(y_{n}, x_{n}\right)=F^{n}\left(y_{0}, x_{0}\right)=F\left(F^{n-1}\left(y_{0}, x_{0}\right)\right.$, $\left.F^{n-1}\left(x_{0}, y_{0}\right)\right)$ for each $n=0,1,2, \ldots$.
If $x_{n}=x_{n+1}$ and $y_{n}=y_{n+1}$ for some $n$, then $\left(x_{n}, y_{n}\right)$ is a coupled fixed point of $F$. So, without loss of generality, we assume that $x_{n} \neq x_{n+1}$ or $y_{n} \neq y_{n+1}$ for all $n$.

Now using (9), we have

$$
\begin{array}{rl}
T( & \frac{\left.d\left(x_{n+1}, y_{n+1}\right), F\left(x_{n}, y_{n}\right)\right)+d\left(F\left(y_{n+1}, x_{n+1}\right), F\left(y_{n}, x_{n}\right)\right)}{2}, \\
& \frac{d\left(x_{n+1}, x_{n}\right)+d\left(y_{n+1}, y_{n}\right)}{2}, \\
& \frac{d\left(x_{n+1}, F\left(x_{n+1}, y_{n+1}\right)\right)+d\left(y_{n+1}, F\left(y_{n+1}, x_{n+1}\right)\right)}{2}, \\
& \frac{d\left(x_{n}, F\left(x_{n}, y_{n}\right)\right)+d\left(y_{n}, F\left(y_{n}, x_{n}\right)\right)}{2}, \\
& \frac{d\left(x_{n+1}, F\left(x_{n}, y_{n}\right)\right)+d\left(y_{n+1}, F\left(y_{n}, x_{n}\right)\right)}{2}, \\
& \left.\frac{d\left(x_{n}, F\left(x_{n+1}, y_{n+1}\right)\right)+d\left(y_{n}, F\left(y_{n+1}, x_{n+1}\right)\right)}{2}\right) \leq 0 \\
& \frac{d\left(x_{n+2}, x_{n+1}\right)+d\left(y_{n+2}, y_{n+1}\right)}{2}, \\
& \frac{d\left(x_{n+1}, x_{n}\right)+d\left(y_{n+1}, y_{n}\right)}{2}, \\
& \frac{d\left(x_{n+1}, x_{n+2}\right)+d\left(y_{n+1}, y_{n+2}\right)}{2}, \\
& \frac{d\left(x_{n}, x_{n+1}\right)+d\left(y_{n}, y_{n+1}\right)}{2}, \\
2 & d\left(y_{n+1}, y_{n+1}\right) \\
2 &
\end{array}
$$

Since $T$ is nonincreasing in 6 th variable, using triangle inequality, we get

$$
\begin{aligned}
& T\left(\frac{d\left(x_{n+2}, x_{n+1}\right)+d\left(y_{n+2}, y_{n+1}\right)}{2},\right. \\
& \frac{d\left(x_{n+1}, x_{n}\right)+d\left(y_{n+1}, y_{n}\right)}{2}, \\
& \quad \frac{d\left(x_{n+1}, x_{n+2}\right)+d\left(y_{n+1}, y_{n+2}\right)}{2}, \\
& \quad \frac{d\left(x_{n}, x_{n+1}\right)+d\left(y_{n}, y_{n+1}\right)}{2}, 0, \\
& \quad\left(d\left(x_{n}, x_{n+1}\right)+d\left(x_{n+1}, x_{n+2}\right)\right. \\
& \left.\quad+d\left(y_{n}, y_{n+1}\right)+d\left(y_{n+1}, y_{n+2}\right)\right) \\
& \left.\quad \times(2)^{-1}\right) \leq 0 .
\end{aligned}
$$


From $\left(T_{2}\right)$, there exists $f: R_{+} \rightarrow R_{+}, f(t)<t$ for $t>0$ such that

$$
\begin{aligned}
\delta_{n+1} & =\frac{d\left(x_{n+1}, x_{n+2}\right)+d\left(y_{n+1}, y_{n+2}\right)}{2} \\
& \leq f\left(\frac{d\left(x_{n+1}, x_{n}\right)+d\left(y_{n+1}, y_{n}\right)}{2}\right)=f\left(\delta_{n}\right) .
\end{aligned}
$$

Then $\delta_{n} \neq 0$ for all $n$. Hence, from (14), we have

$$
\delta_{n+1} \leq f\left(\delta_{n}\right)<\delta_{n}, \quad \forall n
$$

Therefore $\left\{\delta_{n}\right\}$ is strictly decreasing sequence of positive reals. Hence there exists $\delta \geq 0$ such that

$$
\lim _{n \rightarrow \infty} \delta_{n}=\lim _{n \rightarrow \infty} \frac{d\left(x_{n+1}, x_{n+2}\right)+d\left(y_{n+1}, y_{n+2}\right)}{2}=\delta .
$$

We show that $\delta=0$. Suppose that $\delta>0$. On letting $n \rightarrow \infty$ in (13), we get

$$
T(\delta, \delta, \delta, \delta, 0,2 \delta) \leq 0
$$

Then from $\left(T_{2}\right)$, we have $\delta \leq f(\delta)$, a contradiction. Hence $\delta=0$.

$$
\text { That is, } \lim _{n \rightarrow \infty} \frac{d\left(x_{n+1}, x_{n+2}\right)+d\left(y_{n+1}, y_{n+2}\right)}{2}=0 \text {. }
$$

Now we show that $\left\{x_{n}\right\}$ and $\left\{y_{n}\right\}$ are Cauchy sequences in $X$. Suppose that at least one of $\left\{x_{n}\right\}$ or $\left\{y_{n}\right\}$ is not Cauchy. Then there exist $\epsilon>0$ and sequences of positive integers $\{m(k)\}$ and $\{n(k)\}$ with $m(k) \geq n(k)>k$ such that

$$
r_{k}=d\left(x_{m(k)}, x_{n(k)}\right)+d\left(y_{m(k)}, y_{n(k)}\right) \geq \epsilon .
$$

Now choose $m(k)$ the least positive integer such that (19) holds. Then

$$
d\left(x_{m(k)-1}, x_{n(k)}\right)+d\left(y_{m(k)-1}, y_{n(k)}\right)<\epsilon .
$$

Now

$$
\begin{aligned}
\epsilon \leq & r_{k}=d\left(x_{m(k)}, x_{n(k)}\right)+d\left(y_{m(k)}, y_{n(k)}\right) \\
\leq & d\left(x_{m(k)}, x_{m(k)-1}\right)+d\left(x_{m(k)-1}, x_{n(k)}\right) \\
& +d\left(y_{m(k)}, y_{m(k)-1}\right)+d\left(y_{m(k)-1}, y_{n(k)}\right) \\
& <d\left(x_{m(k)}, x_{m(k)-1}\right)+d\left(y_{m(k)}, y_{m(k)-1}\right)+\epsilon .
\end{aligned}
$$

On taking limit supremum as $k \rightarrow \infty$, we get

$$
\epsilon \leq \limsup _{k \rightarrow \infty} r_{k}=\limsup _{k \rightarrow \infty} d\left(x_{m(k)}, x_{n(k)}\right)+d\left(y_{m(k)}, y_{n(k)}\right) \leq \epsilon .
$$

Hence

$$
\limsup _{k \rightarrow \infty} r_{k}=\limsup _{k \rightarrow \infty} d\left(x_{m(k)}, x_{n(k)}\right)+d\left(y_{m(k)}, y_{n(k)}\right)=\epsilon .
$$

Similarly, we can show that

$$
\liminf _{k \rightarrow \infty} r_{k}=\liminf _{k \rightarrow \infty} d\left(x_{m(k)}, x_{n(k)}\right)+d\left(y_{m(k)}, y_{n(k)}\right)=\epsilon .
$$

Hence

$$
\lim _{k \rightarrow \infty} r_{k}=\lim _{k \rightarrow \infty} d\left(x_{m(k)}, x_{n(k)}\right)+d\left(y_{m(k)}, y_{n(k)}\right)=\epsilon
$$

Now

$$
\begin{aligned}
\epsilon \leq & r_{k}=d\left(x_{m(k)}, x_{n(k)}\right)+d\left(y_{m(k)}, y_{n(k)}\right) \\
\leq & d\left(x_{m(k)}, x_{m(k)+1}\right)+d\left(x_{m(k)+1}, x_{n(k)+1}\right) \\
& +d\left(x_{n(k)+1}, x_{n(k)}\right)+d\left(y_{m(k)}, y_{m(k)+1}\right) \\
& +d\left(y_{m(k)+1}, y_{n(k)+1}\right)+d\left(y_{n(k)+1}, y_{n(k)}\right) .
\end{aligned}
$$

Since $m(k) \geq n(k)$, we have $x_{m(k)} \geq x_{n(k)}$ and $y_{m(k)} \preceq y_{n(k)}$ for each $k$. Hence, from (9), we get

$$
\begin{aligned}
T( & d\left(F\left(x_{m(k)}, y_{m(k)}\right), F\left(x_{n(k)}, y_{n(k)}\right)\right) \\
& \left.+d\left(F\left(y_{m(k)}, x_{m(k)}\right), F\left(y_{n(k)}, x_{n(k)}\right)\right)\right) \\
& \times(2)^{-1}, \\
& \frac{d\left(x_{m(k)}, x_{n(k)}\right)+d\left(y_{m(k)}, y_{n(k)}\right)}{2}, \\
& \frac{d\left(x_{m(k)}, F\left(x_{m(k)}, y_{m(k)}\right)\right)+d\left(y_{m(k)}, F\left(y_{m(k)}, x_{m(k)}\right)\right)}{2}, \\
& \frac{d\left(x_{n(k)}, F\left(x_{n(k)}, y_{n(k)}\right)\right)+d\left(y_{n(k)}, F\left(y_{n(k)}, x_{n(k)}\right)\right)}{2}, \\
& \frac{d\left(x_{m(k)}, F\left(x_{n(k)}, y_{n(k)}\right)\right)+d\left(y_{m(k)}, F\left(y_{n(k)}, x_{n(k)}\right)\right)}{2}, \\
& \left.\frac{d\left(x_{n(k)}, F\left(x_{m(k)}, y_{m(k)}\right)\right)+d\left(y_{n(k)}, F\left(y_{m(k)}, x_{m(k)}\right)\right)}{2}\right)
\end{aligned}
$$

$\leq 0$,

$$
\begin{gathered}
T\left(\frac{d\left(x_{m(k)+1}, x_{n(k)+1}\right)+d\left(y_{m(k)+1}, y_{n(k)+1}\right)}{2},\right. \\
\frac{d\left(x_{m(k)}, x_{n(k)}\right)+d\left(y_{m(k)}, y_{n(k)}\right)}{2}, \\
\frac{d\left(x_{m(k)}, x_{m(k)+1}\right)+d\left(y_{m(k)}, y_{m(k)+1}\right)}{2}, \\
\frac{d\left(x_{n(k)}, x_{n(k)+1}\right)+d\left(y_{n(k)}, y_{n(k)+1}\right)}{2},
\end{gathered}
$$




$$
\begin{aligned}
& \frac{d\left(x_{m(k)}, x_{n(k)+1}\right)+d\left(y_{m(k)}, y_{n(k)+1}\right)}{2}, \\
& \left.\frac{d\left(x_{n(k)}, x_{m(k)+1}\right)+d\left(y_{n(k)}, y_{m(k)+1}\right)}{2}\right) \leq 0 .
\end{aligned}
$$

Since $T$ is nonincreasing in 5 th and 6 th variable, using triangle inequality, we get

$$
\begin{aligned}
& T\left(\frac{d\left(x_{m(k)+1}, x_{n(k)+1}\right)+d\left(y_{m(k)+1}, y_{n(k)+1}\right)}{2},\right. \\
& \quad \frac{d\left(x_{m(k)}, x_{n(k)}\right)+d\left(y_{m(k)}, y_{n(k)}\right)}{2}, \\
& \quad \frac{d\left(x_{m(k)}, x_{m(k)+1}\right)+d\left(y_{m(k)}, y_{m(k)+1}\right)}{2}, \\
& \quad \frac{\left(d\left(x_{n(k)}, x_{n(k)+1}\right)+d\left(y_{n(k)}, y_{n(k)+1}\right)\right.}{2}, \\
& \left.\quad+d\left(y_{m(k)}, y_{m(k)+1}\right)+d\left(y_{m(k)+1}, y_{n(k)+1}\right)\right) \\
& \quad \times(2)^{-1}, \\
& \left(d\left(x_{n(k)}, x_{n(k)+1}\right)+d\left(x_{n(k)+1}, x_{m(k)+1}\right)\right. \\
& \left.\quad+d\left(y_{n(k)}, y_{n(k)+1}\right)+d\left(y_{n(k)+1}, y_{m(k)+1}\right)\right) \\
& \left.\times(2)^{-1}\right) \leq 0 .
\end{aligned}
$$

On letting $k \rightarrow \infty$, we get

$$
\begin{aligned}
& T\left(\lim _{k \rightarrow \infty} \frac{d\left(x_{m(k)+1}, x_{n(k)+1}\right)+d\left(y_{m(k)+1}, y_{n(k)+1}\right)}{2},\right. \\
& \left.\quad \frac{\epsilon}{2}, 0,0, \frac{\epsilon}{2}, \frac{\epsilon}{2}\right) \leq 0 .
\end{aligned}
$$

From $\left(T_{2}\right)$, we get

$$
\lim _{k \rightarrow \infty} \frac{d\left(x_{m(k)+1}, x_{n(k)+1}\right)+d\left(y_{m(k)+1}, y_{n(k)+1}\right)}{2} \leq f\left(\frac{\epsilon}{2}\right) .
$$

Now on letting $k \rightarrow \infty$, in (26), we get

$$
\begin{array}{r}
\epsilon \leq \lim _{k \rightarrow \infty}\left[d\left(x_{m(k)}, x_{m(k)+1}\right)+d\left(x_{m(k)+1}, x_{n(k)+1}\right)\right. \\
+d\left(x_{n(k)+1}, x_{n(k)}\right)+d\left(y_{m(k)}, y_{m(k)+1}\right) \\
\left.+d\left(y_{m(k)+1}, y_{n(k)+1}\right)+d\left(y_{n(k)+1}, y_{n(k)}\right)\right] \\
=\lim _{k \rightarrow \infty}\left[d\left(x_{m(k)+1}, x_{n(k)+1}\right)+d\left(y_{m(k)+1}, y_{n(k)+1}\right)\right] .
\end{array}
$$

Hence

$$
\begin{aligned}
\frac{\epsilon}{2} & \leq \lim _{k \rightarrow \infty} \frac{d\left(x_{m(k)+1}, x_{n(k)+1}\right)+d\left(y_{m(k)+1}, y_{n(k)+1}\right)}{2} \\
& \leq f\left(\frac{\epsilon}{2}\right), \text { a contradiction. }
\end{aligned}
$$

Therefore $\left\{x_{n}\right\}$ and $\left\{y_{n}\right\}$ are Cauchy sequences in $X$. Since $X$ is complete there exists $x, y \in X$ with $\lim _{n \rightarrow \infty} x_{n}=x$ and $\lim _{n \rightarrow \infty} y_{n}=y$.

Suppose that $F$ is continuous. Then

$$
\begin{aligned}
& x=\lim _{n \rightarrow \infty} x_{n+1}=\lim _{n \rightarrow \infty} F\left(x_{n}, y_{n}\right)=F(x, y), \\
& y=\lim _{n \rightarrow \infty} y_{n+1}=\lim _{n \rightarrow \infty} F\left(y_{n}, x_{n}\right)=F(y, x) .
\end{aligned}
$$

Therefore $(x, y)$ is a coupled fixed point of $F$.

Now suppose that conditions (b) hold. Hence, we have $x_{n} \preceq x$ for all $n$ and $y \preceq y_{n}$ for all $n$.

Now from (9), we have

$$
\begin{aligned}
& T\left(\frac{d\left(F(x, y), F\left(x_{n}, y_{n}\right)\right)+d\left(F(y, x), F\left(y_{n}, x_{n}\right)\right)}{2},\right. \\
& \quad \frac{d\left(x, x_{n}\right)+d\left(y, y_{n}\right)}{2}, \frac{d(x, F(x, y))+d(y, F(y, x))}{2}, \\
& \quad \frac{d\left(x_{n}, F\left(x_{n}, y_{n}\right)\right)+d\left(y_{n}, F\left(y_{n}, x_{n}\right)\right)}{2}, \\
& \quad \frac{d\left(x, F\left(x_{n}, y_{n}\right)\right)+d\left(y, F\left(y_{n}, x_{n}\right)\right)}{2}, \\
& \left.\quad \frac{d\left(x_{n}, F(x, y)\right)+d\left(y_{n}, F(y, x)\right)}{2}\right) \leq 0 .
\end{aligned}
$$

On letting $n \rightarrow \infty$, we get

$$
\begin{gathered}
T\left(\frac{d(F(x, y), x)+d(F(y, x), y)}{2}, 0,\right. \\
\frac{d(x, F(x, y))+d(y, F(y, x))}{2}, 0,0, \\
\left.\frac{d(x, F(x, y))+d(y, F(y, x))}{2}\right) \leq 0 .
\end{gathered}
$$

If $d(F(x, y), x)+d(F(y, x), y)=0$, then $(x, y)$ is a coupled fixed point of $F$.

If $d(F(x, y), x)+d(F(y, x), y)>0$, then (35) yields a contradiction to $\left(T_{3}\right)$. Hence $F(x, y)=x$ and $F(y, x)=y$. Therefore $(x, y)$ is a coupled fixed point of $F$.

Theorem 16 does not guarantee the uniqueness of coupled fixed point of $F$. The following example supports this assertion.

Example 17. Let $X=\{2,4,6,8\}$ with the usual metric. We define a partial order $\preceq$ on $X$ as follows:

$$
\preceq=\{(2,2),(4,4),(6,6),(8,8),(2,8)\} .
$$


We define $T:\left(R_{+}\right)^{6} \rightarrow R$ by $T\left(t_{1}, \ldots, t_{6}\right)=t_{1}-\alpha\left(t_{4} t_{5} t_{6} /\right.$ $\left.\left(t_{2}+t_{3}+1\right)\right), t_{1}, t_{2}, \ldots, t_{6} \geq 0$ and $\alpha=15 / 16$. Then $T \in \mathcal{S}$ (Example 11).

Let

$$
\begin{gathered}
A=\{(2,2),(2,8),(4,2),(4,4),(4,8), \\
(6,2),(6,8),(8,2),(8,8)\} \\
B=\{(2,4),(8,4),(8,6)\}, \\
C=\{(2,6),(4,6),(6,4),(6,6)\} .
\end{gathered}
$$

We define $F: X \times X \rightarrow X$ by

$$
F(x, y)= \begin{cases}4, & \text { if }(x, y) \in A \\ 8, & \text { if }(x, y) \in B \\ 2, & \text { if }(x, y) \in C\end{cases}
$$

Clearly $F$ satisfies the mixed monotone property. We choose $\left(x_{0}, y_{0}\right)=(2,4)$. Then $F\left(x_{0}, y_{0}\right)=8$ and $F\left(y_{0}, x_{0}\right)=4$ so that $x_{0} \preceq F\left(x_{0}, y_{0}\right)$ and $y_{0} \succeq F\left(y_{0}, x_{0}\right)$. The elements $(x, y)$, $(u, v)$ in $X \times X$ such that $x \geq u$ and $y \preceq v$ are the following: $(2,2),(2,2) ;(2,2),(2,8) ;(2,4),(2,4) ;(2,6),(2,6) ;(2,8)$, $(2,8) ;(4,2),(4,2) ;(4,2),(4,8) ;(4,4),(4,4) ;(4,6),(4,6)$; $(4,8),(4,8) ;(6,2),(6,2) ;(6,2),(6,8) ;(6,4),(6,4) ;(6,6)$, $(6,6) ;(6,8),(6,8) ;(8,2),(2,2) ;(8,2),(2,8) ;(8,2),(8,2)$; $(8,2),(8,8) ;(8,4),(2,4) ;(8,4),(8,4) ;(8,6),(2,6) ;(8,6)$, $(8,6) ;(8,8),(2,8) ;(8,8),(8,8)$. We now verify inequality $(9)$ for the above pairs.

Case i. $(x, y),(u, v) \in X \times X$ such that $(x, y),(u, v),(y, x)$, $(v, u)$ are either in $A$ or $B$ or $C$ or $(x, y)=(u, v)$.

In this case $(d(F(x, y), F(u, v))+d(F(y, x), F(v, u))) / 2=0$ so that inequality (9) holds trivially.

Case ii. $(x, y)=(6,2),(u, v)=(6,8)$ or $(x, y)=(6,8),(u, v)=$ $(2,6)$.

In this case

$$
\begin{aligned}
& \frac{d(F(x, y), F(u, v))+d(F(y, x), F(v, u))}{2} \\
& =3=\frac{15}{16} \frac{16}{5}=\frac{15}{16} \\
& \times\left(\frac{d(u, F(u, v))+d(v, F(v, u))}{2}\right) \\
& \times\left(\frac{d(x, F(u, v))+d(y, F(v, u))}{2}\right) \\
& \times\left(\frac{d(u, F(x, y))+d(v, F(y, x))}{2}\right) \\
& \times\left(\frac{d(x, u)+d(y, v)}{2}\right. \\
& \left.+\frac{d(x, F(x, y))+d(y, F(y, x))}{2}+1\right)^{-1} .
\end{aligned}
$$

Hence inequality (9) holds. Therefore all the hypotheses of Theorem 16 hold and $(8,4)$ and $(4,4)$ are two coupled fixed points of $F$.

In the following theorem, we prove the uniqueness of coupled fixed points by using an implicit relation in $\delta^{\prime}$.

We define a partial order $\preceq_{1}$ on $X \times X$ by $(x, y) \preceq_{1}(u, v)$ if $x \preceq u, y \geq v$.

Theorem 18. In addition to the hypotheses of Theorem 16 with $T \in \mathcal{S}^{\prime}$ suppose that the following condition holds:

for any $(x, y)$ and $(u, v)$ in $X \times X$

there exists $(z, t) \in X \times X$ that is comparable with

$$
(x, y) \text { and }(u, v) \text {. }
$$

Then F has a unique coupled fixed point.

Proof. With the hypotheses of Theorem 16, $F$ has a coupled fixed point $(x, y)$, that is, $x=F(x, y)$ and $y=F(y, x)$. Suppose that if possible there exists $(u, v) \in X \times X$ such that $u=F(u, v)$ and $v=F(v, u)$. We show that $x=u$ and $y=v$.

Case i. $(x, y)$ and $(u, v)$ are comparable.

Without loss of generality, we assume that $(u, v) \preceq_{1}(x, y)$. That is, $x \geq u$ and $y \preceq v$.

Suppose that either $x \neq u$ or $y \neq v$. Then

$$
\begin{aligned}
T( & \frac{d(F(x, y), F(u, v))+d(F(y, x), F(v, u))}{2}, \\
& \frac{d(x, u)+d(y, v)}{2}, \\
& \frac{d(x, F(x, y))+d(y, F(y, x))}{2}, \\
& \frac{d(u, F(u, v))+d(v, F(v, u))}{2}, \\
& \left.\frac{d(u, F(x, y))+d(v, F(y, x))}{2}\right) \leq 0, \\
T\left(\frac{d(x, u)+d(y, v)}{2}, \frac{d(x, u)+d(y, v)}{2}, 0,0,\right. & \left.\frac{d(x, u)+d(y, v)}{2}, \frac{d(u, x)+d(v, y)}{2}\right) \leq 0 .
\end{aligned}
$$

Then, from $\left(T_{2}^{\prime}\right)$, there exists $f: R_{+} \rightarrow R_{+}, f(t)<t$ for $t>0$ such that

$$
\frac{d(x, u)+d(y, v)}{2} \leq f\left(\frac{d(x, u)+d(y, v)}{2}\right),
$$

a contradiction. Hence $x=u$ and $y=v$. 
Case ii. There exists $(z, t) \in X \times X$ which is comparable with $(x, y)$ and $(u, v)$.

Without loss of generality, we assume that $(x, y) \preceq_{1}(z, t)$ and $(u, v) \preceq_{1}(z, t)$, that is, $z \succeq x, t \preceq y$ and $z \succeq u, t \preceq v$.

We denote $F^{n+1}(x, y)=F\left(F^{n}(x, y), F^{n}(y, x)\right)$ for each $x, y \in X$.

Since $F$ has mixed monotone property, we have

$$
\begin{array}{ll}
F(x, y) \preceq F(z, t), & F(u, v) \preceq F(z, t), \\
F(y, x) \succeq F(t, z), & F(v, u) \succeq F(t, z) .
\end{array}
$$

Now suppose that

$$
\begin{aligned}
F^{n}(x, y) & \leq F^{n}(z, t), \quad F^{n}(u, v) \preceq F^{n}(z, t), \\
F^{n}(y, x) & \succeq F^{n}(t, z), \quad F^{n}(v, u) \geq F^{n}(t, z) \\
& \text { for some } n . \\
F^{n+1}(x, y) & =F\left(F^{n}(x, y), F^{n}(y, x)\right) \\
& \leq F\left(F^{n}(z, t), F^{n}(t, z)\right)=F^{n+1}(z, t), \\
F^{n+1}(u, v) & =F\left(F^{n}(u, v), F^{n}(v, u)\right) \\
& \preceq F\left(F^{n}(z, t), F^{n}(t, z)\right)=F^{n+1}(z, t), \\
F^{n+1}(y, x) & =F\left(F^{n}(y, x), F^{n}(x, y)\right) \\
& \geq F\left(F^{n}(t, z), F^{n}(z, t)\right)=F^{n+1}(t, z), \\
F^{n+1}(v, u) & =F\left(F^{n}(v, u), F^{n}(u, v)\right) \\
& \geq F\left(F^{n}(t, z), F^{n}(z, t)\right)=F^{n+1}(t, z) .
\end{aligned}
$$

Hence by mathematical induction,

$$
\begin{array}{rlrl}
F^{n}(x, y) & \preceq F^{n}(z, t), & F^{n}(u, v) & \leq F^{n}(z, t), \\
F^{n}(y, x) & \succeq F^{n}(t, z), & F^{n}(v, u) & \geq F^{n}(t, z) \\
& \text { for each } n=0,1,2, \ldots
\end{array}
$$

We now show that

$$
\lim _{n \rightarrow \infty} F^{n}(z, t)=x, \quad \lim _{n \rightarrow \infty} F^{n}(t, z)=y .
$$

Using (9), we get

$$
\begin{aligned}
T((d & \left(F\left(F^{n}(z, t), F^{n}(t, z)\right), F(x, y)\right) \\
& \left.+d\left(F\left(F^{n}(t, z), F^{n}(z, t)\right), F(y, x)\right)\right) \\
& \times(2)^{-1}, \\
& \frac{d\left(F^{n}(z, t), x\right)+d\left(F^{n}(t, z), y\right)}{2} \\
& \left(d\left(F^{n}(z, t), F\left(F^{n}(z, t)\right), F^{n}(t, z)\right)\right. \\
& \left.+d\left(F^{n}(t, z), F\left(F^{n}(t, z), F^{n}(z, t)\right)\right)\right) \\
& \times(2)^{-1}
\end{aligned}
$$

$$
\begin{aligned}
& \frac{d(x, F(x, y))+d(y, F(y, x))}{2}, \\
& \frac{d\left(F^{n}(z, t), x\right)+d\left(F^{n}(t, z), y\right)}{2}, \\
& \quad+d\left(x, F\left(F^{n}(z, t)\right), F^{n}(t, z)\right) \\
& \left.\quad+d\left(y, F\left(F^{n}(t, z), F^{n}(z, t)\right)\right)\right) \\
& \left.\times(2)^{-1}\right) \leq 0 .
\end{aligned}
$$

This implies

$$
\begin{aligned}
& T\left(\frac{d\left(F^{n+1}(z, t), x\right)+d\left(F^{n+1}(t, z), y\right)}{2},\right. \\
& \quad \frac{d\left(F^{n+1}(t, z), x\right)+d\left(F^{n+1}(z, t), y\right)}{2}, \\
& \\
& \quad \frac{d\left(F^{n}(z, t), F^{n+1}(z, t)\right)+d\left(F^{n}(t, z), F^{n+1}(t, z)\right)}{2}, 0, \\
& \left.\quad \frac{d\left(x, F^{n+1}(z, t)\right)+d\left(y, F^{n+1}(t, z)\right)}{2}\right) \leq 0 .
\end{aligned}
$$

Since $T$ is nonincreasing in $3 r d$ variable, using triangle inequality, we get

$$
\begin{gathered}
T\left(\frac{d\left(F^{n+1}(z, t), x\right)+d\left(F^{n+1}(t, z), y\right)}{2},\right. \\
\frac{d\left(F^{n+1}(t, z), x\right)+d\left(F^{n+1}(z, t), y\right)}{2}, \\
\left.\quad+d\left(F^{n}(t, z), y\right)+d\left(y, F^{n+1}(t, z)\right)\right) \\
\quad \times(2)^{-1}, 0, \\
\frac{d\left(F^{n}(z, t), x\right)+d\left(F^{n}(t, z), y\right)}{2}, \\
\left.\quad \frac{d\left(x, F^{n+1}(z, t)\right)+d\left(y, F^{n+1}(t, z)\right)}{2}\right) \leq 0 .
\end{gathered}
$$

Therefore, from $\left(T_{2}^{\prime}\right)$, there exists $f: R_{+} \rightarrow R_{+}, f(t)<t$ for $t>0$ such that

$$
\begin{aligned}
& \frac{d\left(F^{n+1}(z, t), x\right)+d\left(F^{n+1}(t, z), y\right)}{2} \\
& \quad \leq f\left(\frac{d\left(F^{n}(z, t), x\right)+d\left(F^{n}(t, z), y\right)}{2}\right) .
\end{aligned}
$$


If $d\left(F^{n}(z, t), x\right)+d\left(F^{n}(t, z), y\right)=0$ for some $n$ then

$$
\begin{aligned}
& F^{n+1}(z, t)=F\left(F^{n}(z, t), F^{n}(t, z)\right)=F(x, y)=x, \\
& F^{n+1}(t, z)=F\left(F^{n}(t, z), F^{n}(z, t)\right)=F(y, x)=y .
\end{aligned}
$$

Hence, $F^{n+k}(z, t)=x$ and $F^{n+k}(t, z)=y$ for $k=0,1,2, \ldots$, that is, $\lim _{n \rightarrow \infty} F^{n}(z, t)=x$ and $\lim _{n \rightarrow \infty} F^{n}(t, z)=y$, so that (46) holds.

Suppose that $d\left(F^{n}(z, t), x\right)+d\left(F^{n}(t, z), y\right)>0$ for all $n$; then, from (49), we get

$$
\begin{aligned}
& \frac{d\left(F^{n+1}(z, t), x\right)+d\left(F^{n+1}(t, z), y\right)}{2} \\
& \quad \leq f\left(\frac{d\left(F^{n}(z, t), x\right)+d\left(F^{n}(t, z), y\right)}{2}\right) \\
& \quad<\frac{d\left(F^{n}(z, t), x\right)+d\left(F^{n}(t, z), y\right)}{2}, \quad \forall n .
\end{aligned}
$$

Therefore $\left\{\left(d\left(F^{n}(z, t), x\right)+d\left(F^{n}(t, z), y\right)\right) / 2\right\}$ is a decreasing sequence of nonnegative real numbers. Thus, there exists $\delta \geq$ 0 such that $\lim _{n \rightarrow \infty}\left(d\left(F^{n}(z, t), x\right)+d\left(F^{n}(t, z), y\right)\right) / 2=\delta$.

If $\delta>0$, then, on letting $n \rightarrow \infty$ in (49), we get $T(\delta, \delta$, $2 \delta, 0, \delta, \delta) \leq 0$.

Then, from $\left(T_{2}^{\prime}\right)$, we have $\delta \leq f(\delta)$, a contradiction. Hence $\delta=0$.

That is, $\lim _{n \rightarrow \infty} \frac{d\left(F^{n}(z, t), x\right)+d\left(F^{n}(t, z), y\right)}{2}=0$.
That is, $\lim _{n \rightarrow \infty} F^{n}(z, t)=x, \quad \lim _{n \rightarrow \infty} F^{n}(t, z)=y$.

Since $(u, v)$ is also a coupled fixed point of $F$, we have

$$
\lim _{n \rightarrow \infty} F^{n}(z, t)=u, \quad \lim _{n \rightarrow \infty} F^{n}(t, z)=v .
$$

Hence, by the uniqueness of limit, we have $x=u$ and $y=$ $v$.

Remark 19. In Example 17, $T$ does not belong to $S^{\prime}$ (Example 15) and condition (40) does not hold and $T$ has two coupled fixed points.

Theorem 20. In addition to the hypotheses of Theorem 16 with $T \in \mathcal{S}^{\prime}$ suppose that $x_{0}$ and $y_{0}$ are comparable. Further, assume that (40) holds. Then $F$ has a unique coupled fixed point $(x, y)$ and $x=y$.

Proof. From the proof of Theorem 16, we have that $F$ has a coupled fixed point say $(x, y)$. That is, $x=F(x, y)$ and $y=$ $F(y, x)$. Suppose that $x_{0} \preceq y_{0}$. We show that

$$
x_{n} \preceq y_{n}, \quad \forall n,
$$

using principle of mathematical induction on $n$, where $x_{n}=$ $F\left(x_{n-1}, y_{n-1}\right)$ and $y_{n}=F\left(y_{n-1}, x_{n-1}\right), n=0,1,2, \ldots$ are the sequences defined in the proof of Theorem 16. Suppose that (55) is true for some $n \geq 0$. Then, by mixed monotone property of $F$,

$$
x_{n+1}=F\left(x_{n}, y_{n}\right) \leq F\left(y_{n}, x_{n}\right)=y_{n+1} .
$$

Thus (55) is true for each $n=1,2,3, \ldots$ Now, from (9), we have

$$
\begin{aligned}
& T\left(\frac{d\left(F\left(F^{n}(x, y), F^{n}(y, x)\right), F\left(F^{n}(y, x), F^{n}(x, y)\right)\right)+d\left(F\left(F^{n}(y, x), F^{n}(x, y)\right), F\left(F^{n}(x, y), F^{n}(y, x)\right)\right)}{2},\right. \\
& \quad \frac{d\left(F^{n}(x, y), F^{n}(y, x)\right)+d\left(F^{n}(y, x), F^{n}(x, y)\right)}{2}, \\
& \quad \frac{d\left(F^{n}(x, y), F\left(F^{n}(x, y), F^{n}(y, x)\right)\right)+d\left(F^{n}(y, x), F\left(F^{n}(y, x), F^{n}(x, y)\right)\right)}{2}, \\
& \quad \frac{d\left(F^{n}(y, x), F\left(F^{n}(y, x), F^{n}(x, y)\right)\right)+d\left(F^{n}(x, y), F\left(F^{n}(x, y), F^{n}(y, x)\right)\right)}{2} \\
& \quad \frac{d\left(F^{n}(x, y), F\left(F^{n}(y, x), F^{n}(x, y)\right)\right)+d\left(F^{n}(y, x), F\left(F^{n}(x, y), F^{n}(y, x)\right)\right)}{2}, \\
& \left.\quad \frac{d\left(F^{n}(y, x), F\left(F^{n}(x, y), F^{n}(y, x)\right)\right)+d\left(F^{n}(x, y), F\left(F^{n}(y, x), F^{n}(x, y)\right)\right)}{2}\right) \leq 0 .
\end{aligned}
$$


This implies

$$
\begin{gathered}
T\left(\frac{d\left(F^{n+1}(x, y), F^{n+1}(y, x)\right)+d\left(F^{n+1}(y, x), F^{n+1}(x, y)\right)}{2},\right. \\
\frac{d\left(F^{n}(x, y), F^{n}(y, x)\right)+d\left(F^{n}(y, x), F^{n}(x, y)\right)}{2}, \\
\\
\frac{d\left(F^{n}(x, y), F^{n+1}(x, y)\right)+d\left(F^{n}(y, x), F^{n+1}(y, x)\right)}{2}, \\
\frac{d\left(F^{n}(y, x), F^{n+1}(y, x)\right)+d\left(F^{n}(x, y), F^{n+1}(x, y)\right)}{2} \\
\left.\quad \frac{d\left(F^{n}(y, x), F^{n+1}(x, y)\right)+d\left(F^{n}(x, y), F^{n+1}(y, x)\right)}{2}\right)
\end{gathered}
$$

$\leq 0$

On letting $n \rightarrow \infty$, we get

$$
T(d(x, y), d(x, y), 0,0, d(x, y), d(x, y)) \leq 0,
$$

a contradiction to $\left(T_{3}^{\prime}\right)$, if $d(x, y)>0$. Hence, $d(x, y)=0$; that is, $x=y$.

Remark 21. Theorem 16 is an extension of Theorem 5 to coupled fixed points with an implicit relation of $S$.

Corollary 22. Let $(X, \preceq)$ be a partially ordered set and suppose that $d$ is a metric on $X$ such that $(X, d)$ is a complete metric space. Let $F: X \times X \rightarrow X$ be a mapping satisfying mixed monotone property and suppose that there exists a continuous map $\psi: R_{+} \rightarrow R_{+}, \psi(t)<t$ for $t>0, \psi(t)=0$ if and only if $t=0$ such that

$$
\begin{aligned}
& \frac{d(F(x, y), F(u, v))+d(F(y, x), F(v, u))}{2} \\
& \quad \leq \frac{d(x, u)+d(y, v)}{2}-\psi\left(\frac{d(x, u)+d(y, v)}{2}\right),
\end{aligned}
$$

for each $x, y, u, v \in X$ with $x \geq u$ and $y \preceq v$.

Suppose that either (a) or (b) of Theorem 16 holds.

If there exist $x_{0}, y_{0} \in X$ such that $x_{0} \preceq F\left(x_{0}, y_{0}\right)$ and $y_{0} \succeq$ $F\left(y_{0}, x_{0}\right)$ then $F$ has a coupled fixed point.

Proof. By defining $T:\left(R_{+}\right)^{6} \rightarrow R$ in $(9)$ as $T\left(t_{1}, \ldots, t_{6}\right)=$ $t_{1}-\left(t_{2}-\psi\left(t_{2}\right)\right), t_{1}, t_{2}, \ldots, t_{6} \geq 0$, we obtain (60). Hence the conclusion follows from Theorem 16 .

Corollary 23. Let $(X, \preceq)$ be a partially ordered set and suppose that $d$ is a metric on $X$ such that $(X, d)$ is a complete metric space. Let $F: X \times X \rightarrow X$ be a mapping satisfying mixed monotone property and suppose that there exists a continuous map $\psi: R_{+} \rightarrow R_{+}, \psi(t)<t$ for $t>0, \psi(t)=0$ if and only if $t=0$ such that

$$
\begin{aligned}
d(F(x, y), F(u, v)) \leq & \frac{d(x, u)+d(y, v)}{2} \\
& -\psi\left(\frac{d(x, u)+d(y, v)}{2}\right),
\end{aligned}
$$

for each $x, y, u, v \in X$ with $x \geq u$ and $y \preceq v$.

Suppose that either (a) or (b) of Theorem 16 holds.

If there exist $x_{0}, y_{0} \in X$ such that $x_{0} \preceq F\left(x_{0}, y_{0}\right)$ and $y_{0} \succeq$ $F\left(y_{0}, x_{0}\right)$ then $F$ has a coupled fixed point.

Proof. From (61), we obtain

$$
\begin{aligned}
d(F(v, u), F(y, x)) \leq & \frac{d(y, v)+d(x, u)}{2} \\
& -\psi\left(\frac{d(y, v)+d(x, u)}{2}\right) .
\end{aligned}
$$

Adding (61) and (62), we obtain inequality (60). Hence the conclusion follows from Corollary 22.

Remark 24. Corollary 23 is another version of Theorem 4 with different $\psi$.

Corollary 25. Let $(X, \preceq)$ be a partially ordered set and suppose that $d$ is a metric on $X$ such that $(X, d)$ is a complete metric space. Let $F: X \times X \rightarrow X$ be a mapping satisfying mixed monotone property and suppose that there exists a continuous $\operatorname{map} \varphi: R_{+} \rightarrow R_{+}, \varphi(t)<t$ for $t>0, \varphi(0)=0$ such that

$$
\begin{aligned}
& \frac{d(F(x, y), F(u, v))+d(F(y, x), F(v, u))}{2} \\
& \leq \varphi\left(\frac{d(x, u)+d(y, v)}{2}\right),
\end{aligned}
$$

for each $x, y, u, v \in X$ with $x \geq u$ and $y \preceq v$.

Suppose that either (a) or (b) of Theorem 16 holds.

If there exist $x_{0}, y_{0} \in X$ such that $x_{0} \preceq F\left(x_{0}, y_{0}\right)$ and $y_{0} \succeq$ $F\left(y_{0}, x_{0}\right)$ then $F$ has a coupled fixed point.

Proof. By defining $T:\left(R_{+}\right)^{6} \rightarrow R$ in $(9)$ as $T\left(t_{1}, \ldots, t_{6}\right)=t_{1}-$ $\varphi\left(t_{2}\right), t_{1}, t_{2}, \ldots, t_{6} \geq 0$, we obtain (63). Hence the conclusion follows from Theorem 16.

Corollary 26. Let $(X, \preceq)$ be a partially ordered set and suppose that $d$ is a metric on $X$ such that $(X, d)$ is a complete metric space. Let $F: X \times X \rightarrow X$ be a mapping satisfying mixed monotone property and suppose that there exists a continuous $\operatorname{map} \varphi: R_{+} \rightarrow R_{+}, \varphi(t)<t$ for $t>0, \varphi(0)=0$ such that

$$
d(F(x, y), F(u, v)) \leq \varphi\left(\frac{d(x, u)+d(y, v)}{2}\right)
$$

for each $x, y, u, v \in X$ with $x \geq u$ and $y \preceq v$. 
Suppose that either (a) or (b) of Theorem 16 holds.

If there exist $x_{0}, y_{0} \in X$ such that $x_{0} \leq F\left(x_{0}, y_{0}\right)$ and $y_{0} \geq$ $F\left(y_{0}, x_{0}\right)$ then $F$ has a coupled fixed point.

Proof. From (64), we obtain

$$
\begin{aligned}
d(F(v, u), F(y, x)) \leq & \frac{d(y, v)+d(x, u)}{2} \\
& -\psi\left(\frac{d(y, v)+d(x, u)}{2}\right) .
\end{aligned}
$$

Adding (64) and (65), we obtain inequality (63). Hence the conclusion follows from Corollary 25.

Remark 27. In addition to the hypotheses of Corollary 26, if $F$ satisfies (40), then $F$ has a unique coupled fixed point. Further, if $x_{0}$ and $y_{0}$ are comparable, then $F$ has a unique coupled fixed point $(x, y)$ and $x=y$. The same is true in respect of Corollaries 22, 23, and 25.

Remark 28. In view of Examples 11, 13, and 14, we obtain new coupled fixed point results for mappings satisfying (7), the implicit relations in Examples 13 and 14, respectively, by using Theorem 16.

Remark 29. We obtain Theorem 3 as a corollary to Theorem 16 by choosing $T\left(t_{1}, t_{2}, \ldots, t_{6}\right)=t_{1}-k t_{2}, 0 \leq k<1$.

Example 30. Let $X=R_{+}$with the usual metric and usual ordering. We define $T: R_{+} \rightarrow R_{+}$by

$$
\begin{array}{r}
T\left(t_{1}, \ldots, t_{6}\right)=t_{1}-\left[a t_{2}+b t_{3}+c t_{4}+d t_{5}+e t_{6}\right], \\
\text { where } a=\frac{2}{3}, b=c=d=e=\frac{1}{18} .
\end{array}
$$

Now we define $F: X \times X \rightarrow X$ by

$$
F(x, y)= \begin{cases}\frac{x-y}{2}, & \text { if } x \geq y \\ 0, & \text { otherwise }\end{cases}
$$

Then $F$ satisfies mixed monotone property. There exists a point $\left(x_{0}, y_{0}\right)=(0,1) \in X \times X$ such that $x_{0} \leq F\left(x_{0}, y_{0}\right)$ and $y_{0} \geq F\left(y_{0}, x_{0}\right)$. We now verify inequality (9) for $x, y, u, v \in X$ with $x \geq u$ and $y \leq v$ in the following cases.

Case i. $x \geq y$ and $u \geq v$.

In this case, $F(x, y)=(x-y) / 2, F(u, v)=(u-v) / 2$, $F(y, x)=0$ and $F(v, u)=0$.
Hence

$$
\begin{aligned}
\frac{d(F(x, y), F(u, v))+d(F(y, x), F(v, u))}{2} & =\frac{1}{2}\left[\frac{(x-u)+(v-y)}{2}\right] \\
< & \frac{2}{3}\left[\frac{(x-u)+(y-v)}{2}\right] \\
& =\frac{2}{3}\left[\frac{d(x, u)+d(y, v)}{2}\right] .
\end{aligned}
$$

Case ii. $x \geq y$ and $u<v$.

In this case, $F(x, y)=(x-y) / 2, F(u, v)=0, F(y, x)=0$ and $F(v, u)=(v-u) / 2$.

The verification is similar as in Case i.

Case iii. $x<y$ and $u \geq v$.

This case does not arise, for we have $x \geq u$ and $y \leq v$, and hence $x \geq u \geq v \geq y>x$, which does not hold.

Case iv. $x<y$ and $u<v$.

In this case, $F(x, y)=0, F(u, v)=0, F(y, x)=(y-x) / 2$ and $F(v, u)=(v-u) / 2$.

The verification is similar as in Case i.

From all the above cases, we get

$$
\begin{aligned}
& \frac{d(F(x, y), F(u, v))+d(F(y, x), F(v, u))}{2} \\
& <\frac{2}{3}\left[\frac{d(x, u)+d(y, v)}{2}\right] \\
& <a \frac{d(x, u)+d(y, v)}{2}+b \frac{d(x, F(x, y))+d(y, F(y, x))}{2} \\
& \quad+c \frac{d(u, F(u, v))+d(v, F(v, u))}{2} \\
& \quad+d \frac{d(x, F(u, v))+d(y, F(v, u))}{2} \\
& \quad+e \frac{d(u, F(x, y))+d(v, F(y, x))}{2} .
\end{aligned}
$$

Thus, $F$ satisfies inequality (9). Hence all the hypotheses of Theorem 16 hold and $(0,0)$ is the unique coupled fixed point of $F$.

Here we observe that (2) fails to hold, for, at $x=1, u=$ $v=y=0$, we have $d(F(x, y), F(u, v))=1 / 2$ and $(d(x, u)+$ $d(y, v)) / 2=1 / 2$. Hence, Theorem 3 is not applicable.

Example 31. Let $X=[0,1]$ with the usual metric and usual ordering. Suppose that $\varphi: R_{+} \rightarrow R_{+}$be defined by

$$
\varphi(t)= \begin{cases}\frac{3}{4} t, & \text { if } 0 \leq t<1 \\ \frac{3}{4} \sqrt{t}, & \text { if } t \geq 1 .\end{cases}
$$


We now define $T: R_{+} \rightarrow R_{+}$by

$$
T\left(t_{1}, \ldots, t_{6}\right)=t_{1}-\varphi\left(\max \left\{t_{2}, \frac{t_{3}}{2}, t_{4}, \frac{1}{2}\left(t_{5}+t_{6}\right)\right\}\right) .
$$

Define $F: X \times X \rightarrow X$ by

$$
F(x, y)= \begin{cases}\frac{x^{2}-y^{2}}{2}, & \text { if } x \geq y \\ 0, & \text { otherwise. }\end{cases}
$$

Then $F$ satisfies mixed monotone property. There exists a point $\left(x_{0}, y_{0}\right)=(0,1) \in X \times X$ such that $x_{0} \preceq F\left(x_{0}, y_{0}\right)$ and $y_{0} \geq F\left(y_{0}, x_{0}\right)$. We now verify inequality (9) for $x, y, u, v \in X$ with $x \geq u$ and $y \leq v$ in the following cases.

Case i. $x \geq y$ and $u \geq v$.

In this case,

$$
\begin{array}{cc}
F(x, y)=\frac{x^{2}-y^{2}}{2}, & F(u, v)=\frac{u^{2}-v^{2}}{2}, \\
F(y, x)=0, & F(v, u)=0 .
\end{array}
$$

Hence

$$
\begin{aligned}
& \frac{d(F(x, y), F(u, v))+d(F(y, x), F(v, u))}{2} \\
& =\frac{1}{2}\left|\frac{\left(x^{2}-y^{2}\right)-\left(u^{2}-v^{2}\right)}{2}\right| \\
& =\frac{1}{2}\left[\frac{\left(x^{2}-u^{2}\right)+\left(v^{2}-y^{2}\right)}{2}\right] \\
& =\frac{1}{2}\left[\frac{(x+u)(x-u)+(v+y)(v-y)}{2}\right] \\
& \leq \frac{1}{2}\left[\frac{2(x-u)+2(v-y)}{2}\right] \\
& <\frac{3}{4} \frac{d(x, u)+d(y, v)}{2} .
\end{aligned}
$$

Case ii. $x \geq y$ and $u<v$.

In this case, $F(x, y)=\left(x^{2}-y^{2}\right) / 2, F(u, v)=0, F(y, x)=$ 0 and $F(v, u)=\left(v^{2}-u^{2}\right) / 2$.

The verification is similar as in Case $\mathrm{i}$.

Case iii. $x<y$ and $u \geq v$.

This case does not arise, for we have $x \geq u$ and $y \leq v$, and hence $x \geq u \geq v \geq y>x$, which does not hold.

Case iv. $x<y$ and $u<v$.

In this case,

$$
\begin{aligned}
& F(x, y)=0, \quad F(u, v)=0, \quad F(y, x)=\frac{y^{2}-x^{2}}{2}, \\
& F(v, u)=\frac{v^{2}-u^{2}}{2} .
\end{aligned}
$$

The verification is similar as in Case $\mathrm{i}$.
From all the above cases, we get

$$
\begin{aligned}
& \frac{d(F(x, y), F(u, v))+d(F(y, x), F(v, u))}{2} \frac{3}{4} \frac{d(x, u)+d(y, v)}{2} \\
& \leq \varphi\left(\operatorname { m a x } \left\{\frac{d(x, u)+d(y, v)}{2},\right.\right. \\
& \frac{1}{2} \frac{d(x, F(x, y))+d(y, F(y, x))}{2}, \\
& \frac{d(u, F(u, v))+d(v, F(v, u))}{2}, \\
& \frac{1}{2}\left[\frac{d(x, F(u, v))+d(y, F(v, u))}{2}\right. \\
&\left.\left.\left.+\frac{d(u, F(x, y))+d(v, F(y, x))}{2}\right]\right\}\right) .
\end{aligned}
$$

Hence $F$ satisfies inequality (9). Hence all the hypotheses of Theorem 16 hold and $(0,0)$ is the unique coupled fixed point of $F$. Here we observe that $F$ satisfies the hypotheses of Theorem 18 and Theorem 20 too.

Remark 32. There is another viewpoint of coupled fixed points; that is, they can be viewed as fixed points of an operator on product spaces like what follows.

Let $F: X \times X \rightarrow X$ have a coupled fixed point $(x, y)$, that is, $F(x, y)=x$ and $F(y, x)=y$. We construct an operator $S: X \times X \rightarrow X \times X$ as

$$
S(x, y)=(F(x, y), F(y, x)) \text {. }
$$

Then $(x, y)$ is a coupled fixed point of $F$ if and only if $(x, y)$ is a fixed point of $S$. But this viewpoint may not be helpful always, a case being the present one. It is not immediately possible to find the characterization of the operator $S$ from the implicit inequality satisfied by $F$. This justifies the study of coupled fixed points as it is traditionally done without an appeal to the product space.

\section{An Application}

In this section, we establish the existence of solution for an integral equation as an application to the above results in Section 3.

Consider the integral equation

$$
\begin{aligned}
x(t)= & \int_{a}^{b}\left(K_{1}(t, s)+K_{2}(t, s)\right)(f(s, x(s))+g(s, x(s))) d s \\
& +h(t), \quad t \in I=[a, b] .
\end{aligned}
$$


Assumption A. Suppose that $K_{1}, K_{2} \in C(I \times I, R), f, g \in C(I \times$ $R, R)$ and $h \in C(I, R)$ satisfy the following assumptions:

(i) $K_{1}(t, s) \geq 0, K_{2}(t, s) \leq 0$ for all $t, s \in[a, b]$;

(ii) there exist $\lambda, \mu>0$ and a nondecreasing mapping $\theta$ : $R_{+} \rightarrow R_{+}$such that, for all $x, y \in I, x \geq y$,

$$
\begin{aligned}
& 0 \leq f(t, x)-f(t, y) \leq \lambda \theta(x-y), \\
& -\mu \theta(x-y) \leq g(t, x)-g(t, y) \leq 0 .
\end{aligned}
$$

(iii) there exists $\varphi: R_{+} \rightarrow R_{+}, \varphi(t)<t$ for $t>0$ such that $\theta(t)=\varphi(t / 2)$ for all $t \geq 0$;

(iv) $\max \{\lambda, \mu\} \sup _{t \in I} \int_{a}^{b}\left(K_{1}(t, s)-K_{2}(t, s)\right) d s \leq 1 / 2$.

As an example for the existence of mappings satisfying (iii), we can consider the following.

For $\theta(x)=x^{2} / 2(x+2)$, there exists $\varphi, \varphi(x)=x^{2} /(x+1)$ such that $\theta(x)=\varphi(x / 2), \varphi(x)<x$ for $x>0$.

Definition 33. An element $(\alpha, \beta) \in C(I, R) \times C(I, R)$ is called a coupled lower and upper solution of the integral equation (78), if $\alpha(t) \leq \beta(t)$ for each $t \in I$ and

$$
\begin{aligned}
\alpha(t) \leq & \int_{a}^{b} K_{1}(t, s)(f(s, \alpha(s))+g(s, \beta(s))) d s \\
& +\int_{a}^{b} K_{2}(t, s)(f(s, \beta(s))+g(s, \alpha(s))) d s \\
& +h(t), \\
\beta(t) \geq & \int_{a}^{b} K_{1}(t, s)(f(s, \beta(s))+g(s, \alpha(s))) d s \\
& +\int_{a}^{b} K_{2}(t, s)(f(s, \alpha(s))+g(s, \beta(s))) d s \\
& +h(t),
\end{aligned}
$$

for each $t \in I=[a, b]$.

Theorem 34. Consider the integral equation (78) with $K_{1}, K_{2} \in C(I \times I, R), f, g \in C(I \times R, R)$ and $h \in C(I, R)$ and suppose that Assumption $A$ holds. If the integral equation (78) has a coupled lower and upper solution, then it has a unique solution in $C(I, R)$.

Proof. Let $X=C(I, R)$. Define an ordering on $X$ as follows:

$$
x \preceq y \Longleftrightarrow x(t) \leq y(t), \quad \forall t \in[a, b], x, y \in C(I, R) .
$$

With this relation, $X$ is a partially ordered set. Define a metric $d$ on $X$ by

$$
d(x, y)=\sup _{t \in I}|x(t)-y(t)|, \quad x, y \in C(I, R) .
$$

Then $(X, d)$ is a complete metric space.
Suppose that $\left\{u_{n}\right\}$ is a nondecreasing sequence in $X$ which converges to $u$ in $X$. Then we have

$$
u_{1}(t) \leq u_{2}(t) \leq \cdots \leq u_{n}(t) \leq \cdots, \quad \forall t \in I .
$$

Therefore $\left\{u_{n}(t)\right\}$ is a nondecreasing sequence of real numbers and converges to $u(t)$. Hence $u_{n}(t) \leq u(t)$ for all $t \in I$ and $n=1,2,3, \ldots$ so that $u_{n} \leq u$ for all $n$.

Similarly, it is easy to see that if $\left\{v_{n}\right\}$ is a nonincreasing sequence in $X$ that converges to $v \in X$ then $v \leq v_{n}$ for all $n$.

Define $F: X \times X \rightarrow X$ by

$$
\begin{aligned}
F(x, y)(t)= & \int_{a}^{b} K_{1}(t, s)(f(s, x(s))+g(s, y(s))) d s \\
& +\int_{a}^{b} K_{2}(t, s)(f(s, y(s))+g(s, x(s))) d s \\
& +h(t), \quad \forall t \in I .
\end{aligned}
$$

Now we show that $F$ satisfies mixed monotone property.

Let $x_{1}, x_{2} \in X, y \in X$ with $x_{1} \preceq x_{2}$. That is, $x_{1}(t) \leq x_{2}(t)$ for all $t \in I$. Then we have

$$
\begin{aligned}
& F\left(x_{1}, y\right)(t)-F_{2}\left(x_{2}, y\right)(t) \\
& =\int_{a}^{b} K_{1}(t, s)\left(f\left(s, x_{1}(s)\right)+g(s, y(s))\right) d s \\
& \quad+\int_{a}^{b} K_{2}(t, s)\left(f(s, y(s))+g\left(s, x_{1}(s)\right)\right) d s \\
& \quad-\int_{a}^{b} K_{1}(t, s)\left(f\left(s, x_{2}(s)\right)+g(s, y(s))\right) d s \\
& \quad+\int_{a}^{b} K_{2}(t, s)\left(f(s, y(s))+g\left(s, x_{2}(s)\right)\right) d s \\
& =\int_{a}^{b} K_{1}(t, s)\left(f\left(s, x_{1}(s)\right)-f\left(s, x_{2}(s)\right)\right) d s \\
& \quad+\int_{a}^{b} K_{2}(t, s)\left(g\left(s, x_{1}(s)\right)-g\left(s, x_{2}(s)\right)\right) d s
\end{aligned}
$$

$\leq 0$, by Assumption A.

Hence $F\left(x_{1}, y\right)(t) \leq F\left(x_{2}, y\right)(t)$ for all $t \in I$. That is, $F\left(x_{1}, y\right) \preceq$ $F\left(x_{2}, y\right)$.

Now let $y_{1} \geq y_{2}$. That is, $y_{1}(t) \geq y_{2}(t)$ for all $t \in I$. Then we have

$$
\begin{aligned}
& F\left(x, y_{1}\right)(t)-F_{2}\left(x, y_{2}\right)(t) \\
& =\int_{a}^{b} K_{1}(t, s)\left(f(s, x(s))+g\left(s, y_{1}(s)\right)\right) d s \\
& \quad+\int_{a}^{b} K_{2}(t, s)\left(f\left(s, y_{1}(s)\right)+g(s, x(s))\right) d s \\
& \quad-\int_{a}^{b} K_{1}(t, s)\left(f(s, x(s))+g\left(s, y_{2}(s)\right)\right) d s
\end{aligned}
$$




$$
\begin{gathered}
\quad+\int_{a}^{b} K_{2}(t, s)\left(f\left(s, y_{2}(s)\right)+g(s, x(s))\right) d s \\
=\int_{a}^{b} K_{1}(t, s)\left(g\left(s, y_{1}(s)\right)-g\left(s, y_{2}(s)\right)\right) d s \\
+\int_{a}^{b} K_{2}(t, s)\left(f\left(s, y_{1}(s)\right)-f\left(s, y_{2}(s)\right)\right) d s
\end{gathered}
$$

$\leq 0$, by Assumption A.

Hence $F\left(x, y_{1}\right)(t) \leq F\left(x, y_{2}\right)(t)$ for all $t \in I$. That is, $F\left(x, y_{1}\right) \preceq$ $F\left(x, y_{2}\right)$. Thus $F$ satisfies mixed monotone property.

Now, we show that inequality (60) is satisfied. Let $x \geq u$ and $y \leq v$; that is, $x(t) \geq u(t)$ and $y(t) \leq v(t)$. Then, we have

$$
\begin{aligned}
& d(F(x, y), F(u, v)) \\
& =\sup _{t \in I}|F(x, y)(t)-F(u, v)(t)| \\
& =\sup _{t \in I} \mid\left(\int_{a}^{b} K_{1}(t, s)(f(s, x(s))+g(s, y(s))) d s\right. \\
& \left.+\int_{a}^{b} K_{2}(t, s)(f(s, y(s))+g(s, x(s))) d s\right) \\
& -\left(\int_{a}^{b} K_{1}(t, s)(f(s, u(s))+g(s, v(s))) d s\right. \\
& \left.+\int_{a}^{b} K_{2}(t, s)(f(s, v(s))+g(s, u(s))) d s\right) \mid \\
& =\sup _{t \in I} \mid \int_{a}^{b} K_{1}(t, s) \\
& \times[(f(s, x(s))-f(s, u(s))) \\
& +(g(s, y(s))-g(s, v(s)))] d s \\
& +\int_{a}^{b} K_{2}(t, s) \\
& \times[(f(s, y(s))-f(s, v(s))) \\
& +(g(s, x(s))-g(s, u(s)))] d s \mid \\
& =\sup _{t \in I} \mid \int_{a}^{b} K_{1}(t, s) \\
& \times[(f(s, x(s))-f(s, u(s))) \\
& -(g(s, v(s))-g(s, y(s)))] d s \\
& -\int_{a}^{b} K_{2}(t, s) \\
& \times[(f(s, v(s))-f(s, y(s))) \\
& -(g(s, x(s))-g(s, u(s)))] d s \mid
\end{aligned}
$$

$$
\begin{aligned}
& \leq \sup _{t \in I} \mid \int_{a}^{b} K_{1}(t, s) \\
& \times[\lambda \theta(x(s)-u(s)) \\
& +\mu \theta(v(s)-y(s))] d s \\
& -\int_{a}^{b} K_{2}(t, s)[\lambda \theta(v(s)-y(s)) \\
& +\mu \theta(x(s)-u(s))] d s \mid \\
& \leq \max \{\lambda, \mu\} \\
& \times \sup _{t \in I} \int_{a}^{b}\left(K_{1}(t, s)-K_{2}(t, s)\right) \\
& \times(\theta((x(s)-u(s)))+\theta(v(s)-y(s))) \\
& \leq \max \{\lambda, \mu\} \\
& \times\left(\sup _{t \in I} \int_{a}^{b}\left(K_{1}(t, s)-K_{2}(t, s)\right)\right) \\
& \times(\theta(d(x, u))+\theta(d(y, v))) \\
& \leq \frac{1}{2}[\theta(d(x, u))+\theta(d(y, v))] \\
& \leq \theta(d(x, u)+d(y, v)) \\
& =\varphi\left(\frac{d(x, u)+d(y, v)}{2}\right) \text {, by Assumption A (iii). }
\end{aligned}
$$

Therefore, for $x \geq u$ and $y \preceq v$, we have

$$
d(F(x, y), F(u, v)) \leq \varphi\left(\frac{d(x, u)+d(y, v)}{2}\right) .
$$

Let $(\alpha, \beta)$ be lower and upper solution of the integral equation (78); then we have $\alpha(t) \leq \beta(t)$ and $\alpha(t) \leq F(\alpha, \beta)(t)$ and $\beta(t) \geq F(\beta, \alpha)(t)$ for all $t \in I$. Hence, $\alpha \leq \beta, \alpha \leq F(\alpha, \beta)$ and $\beta \geq F(\beta, \alpha)$.

Thus $F$ satisfies all the hypotheses of Corollary 22 and hence $F$ has a coupled fixed point.

Also, with the partial ordering $\preceq$ on $X \times X$ defined by

$$
\begin{gathered}
(x, y) \leq(u, v) \Longleftrightarrow x(t) \leq u(t), \\
y(t) \geq v(t), \quad \forall t \in I,
\end{gathered}
$$

we have $(\max \{x, u\}, \min \{y, v\}) \in X \times X$ comparable with $(x, y)$ and $(u, v)$ for each $(x, y),(u, v) \in X \times X$.

Thus $F$ also satisfies condition (40). Hence $F$ has a unique coupled fixed point $(x, y)$ (say). 
Since $\alpha$ and $\beta$ are comparable, by Theorem 18, it follows that $x=y$. Hence

$$
\begin{aligned}
x(t) & \\
= & F(x, x)(t) \\
= & \int_{a}^{b} K_{1}(t, s)(f(s, x(s))+g(s, x(s))) d s \\
& +\int_{a}^{b} K_{2}(t, s)(f(s, x(s))+g(s, x(s))) d s+h(t) \\
= & \int_{a}^{b}\left(K_{1}(t, s)+K_{2}(t, s)\right)(f(s, x(s))+g(s, x(s))) d s \\
& +h(t) .
\end{aligned}
$$

Hence $x(t)$ is the unique solution of the integral equation (78).

The following is an example as an illustration of Theorem 34

Example 35. Let $I=[0,1]$. Let us now consider the integral equation

$$
\begin{aligned}
x(t)= & -\frac{1}{6} \int_{0}^{1} s(t+s)\left(\frac{x}{8} x(s)+\frac{s}{4\left(1+x^{2}(s)\right)}\right) d s \\
& +(a t+b), \quad t \in I,
\end{aligned}
$$

where $a=145 / 288+(\log 5) / 2-(\log 2) / 16$ and $b=65 / 384-$ $\operatorname{Tan}^{-1}(1 / 2)$.

We transform this equation into Fredholm integral equation of the form (78). That is,

$$
\begin{aligned}
x(t)= & \int_{a}^{b}\left(K_{1}(t, s)+K_{2}(t, s)\right) \\
& \times(f(s, x(s))+g(s, x(s))) d s \\
& +h(t), \quad t \in I=[a, b],
\end{aligned}
$$

where $K_{1}(t, s), K_{2}(t, s): I \times I \rightarrow R, K_{1}(t, s)=(1 / 6)(t+s)$ and $K_{2}(t, s)=-(1 / 3)(t+s)$ for all $t, s \in[0,1], f, g: I \times X \rightarrow X$, $f(t, x)=(t / 8) x$ and $g(t, x)=t / 4\left(1+x^{2}\right), t \in I, x \in X$ and $h: I \rightarrow R, h(t)=a t+b$. Here $K_{1}(t, s) \geq 0$ and $K_{2}(t, s) \leq 0$ for all $t, s \in[0,1]$. It is easy to see that $\sup _{t \in I} \int_{0}^{1}\left(K_{1}(t, s)-\right.$ $\left.K_{2}(t, s)\right) d s=3 / 4$.

Write $X=C(I)$. We define $\theta, \varphi: R_{+} \rightarrow R_{+}$by $\theta(t)=t / 4$ and $\varphi(t)=t / 2$. We choose $\lambda=1 / 2$ and $\mu=3 \sqrt{3} / 8$ so that

$$
\begin{aligned}
& 0 \leq f(t, x)-f(t, y) \leq \lambda \theta(x-y), \\
& -\mu \theta(x-y) \leq g(t, x)-g(t, y) \leq 0,
\end{aligned}
$$

hold. Also, $\max \{\lambda, \mu\} \sup _{t \in I} \int_{a}^{b}\left(K_{1}(t, s)-K_{2}(t, s)\right) d s=3 \sqrt{3} / 8$. $3 / 4=9 \sqrt{3} / 32<1 / 2$.
Hence the conditions (i) to (iv) of Assumption A hold. Then $(\alpha, \beta)$ with $\alpha(t)=t / 2$ and $\beta(t)=t$ is a coupled lower and upper solution of the integral equation (78) and $x(t)=$ $t / 2$ is the unique solution.

\section{Conflict of Interests}

The authors declare that there is no conflict of interests regarding the publication of this paper.

\section{Acknowledgment}

Professor B. S. Choudhury suggested some remarks which are hereby acknowledged.

\section{References}

[1] J. Harjani and K. Sadarangani, "Generalized contractions in partially ordered metric spaces and applications to ordinary differential equations," Nonlinear Analysis: Theory, Methods \& Applications, vol. 72, no. 3-4, pp. 1188-1197, 2010.

[2] J. Harjani and K. Sadarangani, "Fixed point theorems for weakly contractive mappings in partially ordered sets," Nonlinear Analysis: Theory, Methods \& Applications, vol. 71, no. 7-8, pp. 34033410,2009

[3] H. K. Nashine and B. Samet, "Fixed point results for mappings satisfying $(\psi, \varphi)$-weakly contractive condition in partially ordered metric spaces," Nonlinear Analysis, vol. 74, pp. 22012209, 2011.

[4] J. J. Nieto and R. Rodriguez-Lopez, "Contractive mapping theorems in partially ordered sets and applications to ordinary differential equations," Order, vol. 22, no. 3, pp. 223-239, 2005.

[5] J. J. Nieto and R. Rodríguez-López, "Existence and uniqueness of fixed point in partially ordered sets and applications to ordinary differential equations," Acta Mathematica Sinica, vol. 23, no. 12, pp. 2205-2212, 2007.

[6] A. C. Ran and M. C. Reurings, "A fixed point theorem in partially ordered sets and some applications to matrix equations," Proceedings of the American Mathematical Society, vol. 132, no. 5, pp. 1435-1443, 2004.

[7] D. J. Guo and V. Lakshmikantham, "Coupled fixed points of nonlinear operators with applications," Nonlinear Analysis: Theory, Methods \& Applications, vol. 11, no. 5, pp. 623-632, 1987.

[8] T. Gnana Bhaskar and V. Lakshmikantham, "Fixed point theorems in partially ordered metric spaces and applications," Nonlinear Analysis: Theory, Methods \& Applications, vol. 65, no. 7, pp. 1379-1393, 2006.

[9] V. Lakshmikantham and L. B. Ciric, "Coupled fixed point theorems for nonlinear contractions in partially ordered metric spaces," Nonlinear Analysis: Theory, Methods \& Applications, vol. 70, no. 12, pp. 4341-4349, 2009.

[10] B. S. Choudhury and A. Kundu, "A coupled coincidence point result in partially ordered metric spaces for compatible mappings," Nonlinear Analysis. Theory, Methods \& Applications, vol. 73, no. 8, pp. 2524-2531, 2010.

[11] N. V. Luong and N. X. Thuan, "Coupled fixed points in partially ordered metric spaces and application," Nonlinear Analysis. Theory, Methods \& Applications, vol. 74, no. 3, pp. 983-992, 2011.

[12] I. Altun and H. Simsek, "Some fixed point theorems on ordered metric spaces and application," Fixed Point Theory and Applications, vol. 2010, Article ID 621469, 2010. 
[13] V. Popa, "Some fixed point theorems for compatible mappings satisfying an implicit relation," Demonstratio Mathematica, vol. 32, no. 1, pp. 157-163, 1999.

[14] I. Altun and D. Turkoglu, "Some fixed point theorems for weakly compatible multivalued mappings satisfying an implicit relation," Taiwanese Journal of Mathematics, vol. 22, no. 1, pp. 13-21, 2008.

[15] I. Altun and D. Turkoglu, "Some fixed point theorems for weakly compatible mappings satisfying an implicit relation," Taiwanese Journal of Mathematics, vol. 13, no. 4, pp. 1291-1304, 2009.

[16] M. Imdad, S. Kumar, and M. S. Khan, "Remarks on some fixed point theorems satisfying implicit relations," Radovi Matematicki, vol. 11, no. 1, pp. 135-143, 2002.

[17] V. Popa, "A general coincidence theorem for compatible multivalued mappings satisfying an implicit relation," Demonstratio Mathematica, vol. 33, no. 1, pp. 159-164, 2000.

[18] V. Popa and M. Mocanu, "Altering distance and common fixed points under implicit relations," Hacettepe Journal of Mathematics and Statistics, vol. 38, no. 3, pp. 329-337, 2009.

[19] S. Sharma and B. Deshpande, "On compatible mappings satisfying an implicit relation in common fixed point consideration," Tamkang Journal of Mathematics, vol. 33, no. 3, pp. 245-252, 2002.

[20] D. Turkoglu and I. Altun, "A common fixed point theorem for weakly compatible mappings in symmetric spaces satisfying an implicit relation," Sociedad Matematica Mexicana Boletin, vol. 13, no. 1, pp. 195-205, 2007. 


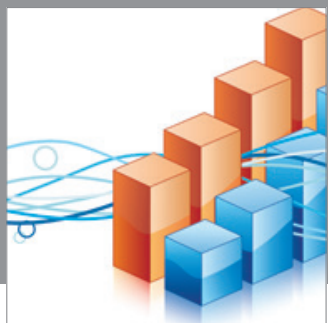

Advances in

Operations Research

mansans

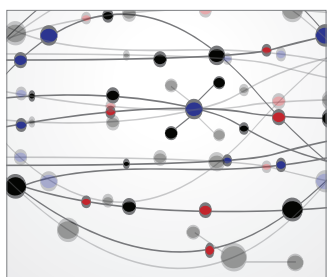

The Scientific World Journal
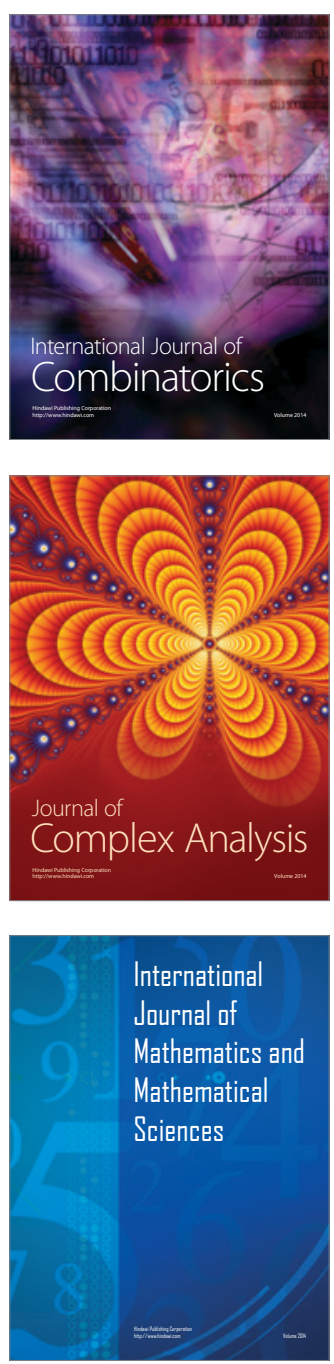
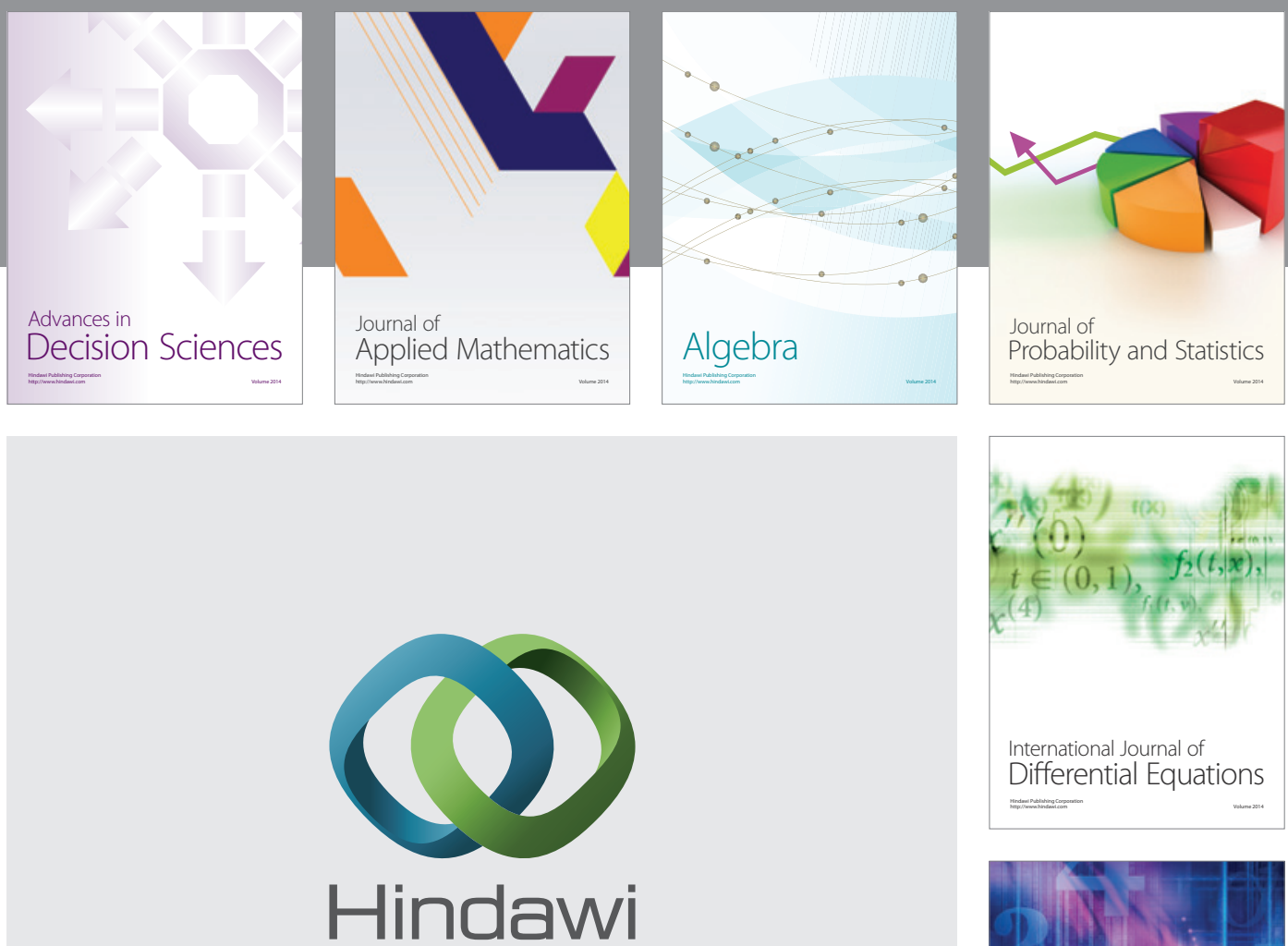

Submit your manuscripts at http://www.hindawi.com
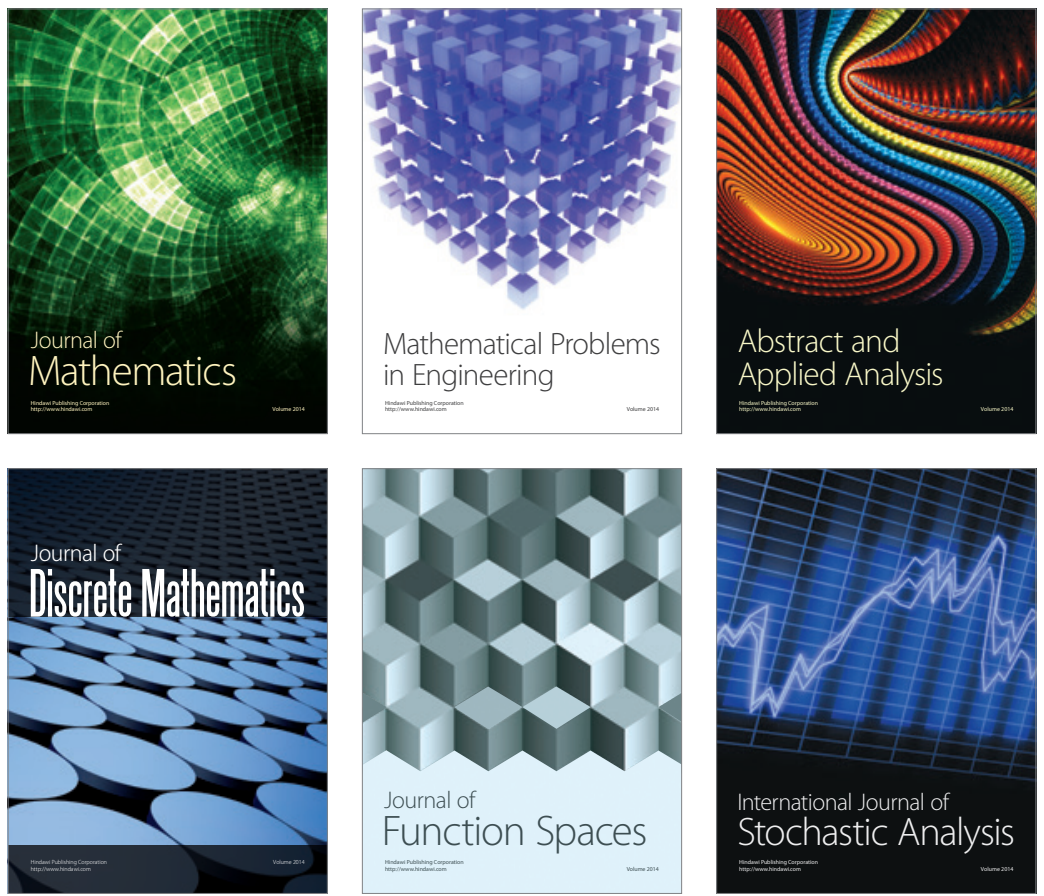

Journal of

Function Spaces

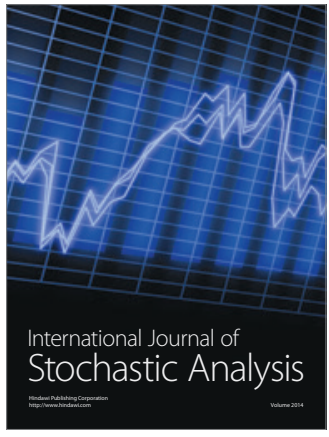

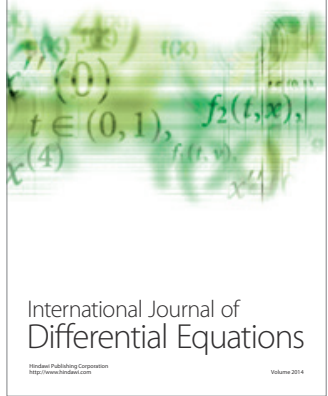
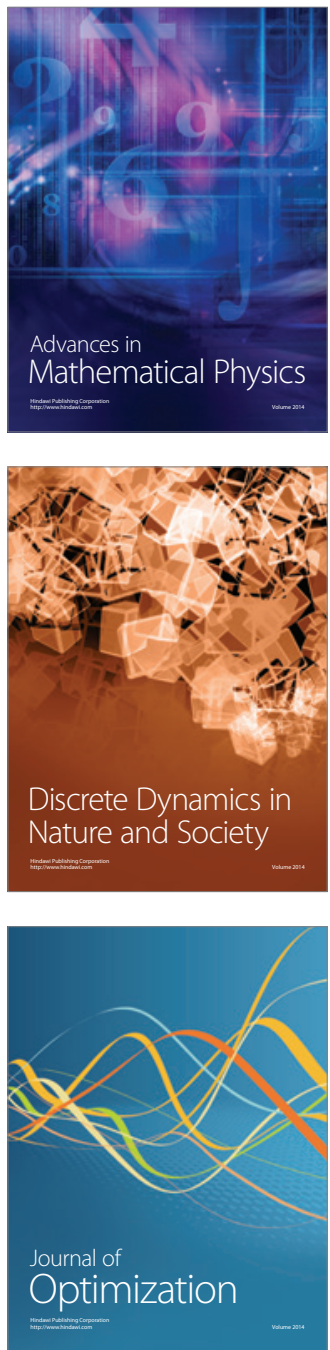